Abstract-This paper proposes a decentralized method for nonlinear control of oscillatory dynamics in power systems. The method is applicable for ensuring both transient stability and small-signal stability. The method uses an optimal control law, which has been derived in the general framework of nonlinear control using normal forms. The model used to derive the control law is the detailed subtransient model of synchronous machines, as recommended by the IEEE. Minimal approximations have been made in either the derivation or the application of the control law. The developed method also requires the application of the dynamic state estimation technique. As the employed control and estimation schemes only need local measurements, the method remains completely decentralized. The method has been demonstrated as an effective tool to prevent blackouts by simulating a major disturbance in a benchmark power system model and its subsequent control using the proposed method.

Index Terms-Decentralized, dynamic state estimation, feedback linearization, lie derivative, nonlinear control, normal form, optimal control, subtransient model, unscented Kalman filtering.

\title{
Decentralized Nonlinear Control for Power Systems Using Normal Forms and Detailed Models
}

\author{
Abhinav Kumar Singh, Member, IEEE, and Bikash C. Pal, Fellow, IEEE
}

\begin{tabular}{|c|c|}
\hline & NOMENCLATURE \\
\hline 0 & Zero matrix (or vector) of appropriate size \\
\hline$\phi$ & $\begin{array}{l}\text { Vector field of state-mapping in normal form trans- } \\
\text { formation }\end{array}$ \\
\hline$B$ & Imaginary part of the bus admittance matrix in p.u. \\
\hline$C(\boldsymbol{x})$ & $\begin{array}{l}\text { Characteristic matrix with }(i, j) \text { element }=L_{\boldsymbol{g}_{j}} \\
L_{\boldsymbol{f}}^{\left(r_{i}-1\right)} h_{i}(\boldsymbol{x})\end{array}$ \\
\hline $\boldsymbol{D}(\boldsymbol{x})$ & Vector $\left[L_{f}^{r_{1}} h_{1}(\boldsymbol{x}) L_{f}^{r_{2}} h_{2}(\boldsymbol{x}) \ldots L_{f}^{r_{m}} h_{m}(\boldsymbol{x})\right]^{T}$ \\
\hline $\boldsymbol{F}$ & Vector of machine-state functions \\
\hline$f$ & Vector of system state functions, $\left[f_{1} f_{2} \ldots f_{n}\right]^{T}$ \\
\hline$G$ & Real part of the bus admittance matrix in p.u. \\
\hline $\boldsymbol{g}_{i}$ & $i$ th vector of input functions for $i=1,2, \ldots, m$ \\
\hline$h$ & Vector of output functions, $\left[h_{1} h_{2} \ldots h_{m}\right]^{T}$ \\
\hline$I$ & Identity matrix of appropriate size \\
\hline $\boldsymbol{I}_{g}$ & Column vector of current injections at system buses \\
\hline
\end{tabular}

Manuscript received December 20, 2015; revised May 24, 2016, September 27, 2016, January 21, 2017, and March 21, 2017; accepted July 2, 2017. This work was supported by the Engineering and Physical Sciences Research Council, U.K., under Grants EP/K036173/1 and EESC-P55251. Paper no. TPWRS01810-2015. (Corresponding author: Abhinav Kumar Singh.)

A. K. Singh is with the School of Engineering, University of Lincoln, Lincoln, LN6 7TS, U.K. (e-mail: asingh@lincoln.ac.uk).

B. C. Pal is with the Control and Power Group, Department of Electrical and Electronic Engineering, Imperial College London, London, SW7 2BT, U.K. (e-mail: b.pal@imperial.ac.uk).

Color versions of one or more of the figures in this paper are available online at http://ieeexplore.ieee.org.

Digital Object Identifier 10.1109/TPWRS.2017.2724022

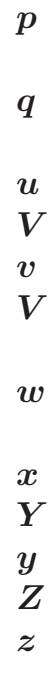

$\delta, \delta_{0}$

$\omega, \omega_{0}$

$\Psi_{1 d}$

$\Psi_{2 q}$

$\theta$

$D$

$E_{d c}^{\prime}$

$E_{d}^{\prime}$

$E_{q}^{\prime}$

$E_{f d}$

$H$

$i, k$

$I_{g}$

$I_{d}, I_{q}$

$j$

$K_{a}$

$K_{d 1}$

$K_{d 2}$

$K_{q 1}$

$K_{q 2}$

$L, M$

$m, n$

$P, Q$

$P_{G}, Q_{G}$ $(n-r) \times m$ matrix with $(j, i)$ element $=L_{\boldsymbol{g}_{i}} \phi_{j+r}$ $\left(\phi^{-1}\right)$

$(n-r) \times 1$ vector with $j$ th element $=L_{\boldsymbol{f}} \phi_{j+r}$ $\left(\phi^{-1}\right)$

Vector of inputs, $\left[u_{1} u_{2} \ldots u_{m}\right]^{T}$

Column vector of terminal voltage of system buses

Vector of inputs in normal form, $\left[v_{1} v_{2} \ldots v_{m}\right]^{T}$

Vector of terminal voltages of all the machines in the system

Internal dynamics' state vector, $\left[\begin{array}{lll}w_{r+1} & w_{r+2} & \ldots\end{array}\right.$ $\left.w_{n}\right]^{T}$

Vector of states, $\left[x_{1} x_{2} \ldots x_{n}\right]^{T}$

Admittance matrix in p.u.

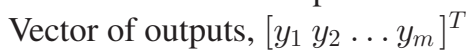

Impedance matrix in p.u.

Vector of linearized states, $\left[z_{1}^{1} \ldots z_{r_{1}}^{1} \ldots z_{1}^{m}\right.$ $\left.\ldots z_{r_{m}}^{m}\right]^{T}$

Rotor angle and its initial operating value, respectively, in rad

Rotor-speed and its synchronous value in $\mathrm{rad} / \mathrm{s}$, respectively

Subtransient emfs due to $d$ axis damper coil in p.u.

Subtransient emfs due to $q$ axis damper coil in p.u.

Bus voltage phase in rad

Rotor damping constant in p.u.

State of the dummy-rotor coil in p.u.

Transient emf due to flux in $q$-axis damper coil in p.u.

Transient emf due to field flux linkages in p.u.

Field excitation voltage in p.u.

Generator inertia constant in $\mathrm{s}$

$i$ th machine (or bus) and $k$ th sample, respectively

Net current injection at a machine bus in p.u.

$d$-axis and $q$-axis stator currents, respectively, in p.u.

$j$ th state or $\sqrt{-1}$, as per context

AVR gain in p.u.

Ratio $\left(X_{d}^{\prime \prime}-X_{l}\right) /\left(X_{d}^{\prime}-X_{l}\right)$

Ratio $\left(X_{d}^{\prime}-X_{d}^{\prime \prime}\right) /\left(X_{d}^{\prime}-X_{l}\right)$

Ratio $\left(X_{q}^{\prime \prime}-X_{l}\right) /\left(X_{q}^{\prime}-X_{l}\right)$

Ratio $\left(X_{q}^{\prime}-X_{q}^{\prime \prime}\right) /\left(X_{q}^{\prime}-X_{l}\right)$

38

40

41

42

43

44

45

46

47

48

49

50

51

52

53

54

55

56

57

Total machines (=total inputs $=$ total outputs) and states, respectively

Net active and reactive power injected at a bus, respectively, in p.u.

Active and reactive power output of a machine, respectively, in p.u. 
$84 r=\sum_{i=1}^{m} r_{i}$, where $r_{i}$ is the relative degree of $y_{i}$

$85 R_{s} \quad$ Armature resistance in p.u.

$86 t \quad$ System time in $\mathrm{s}$

$87 T, T_{0} \quad$ Transpose and the sampling period (in s), respectively

$88 T_{c}, T_{r} \quad$ Time constants for dummy-rotor coil \& AVR filter in

\section{$\mathrm{s}$, respectively}

$T_{e}, T_{m} \quad$ Electrical and mechanical torques, respectively, in p.u.

$T_{d 0}^{\prime}, T_{q 0}^{\prime} \quad d$-axis and $q$-axis transient time constants, respectively, in $\mathrm{s}$

$T_{d 0}^{\prime \prime}, T_{q 0}^{\prime \prime} \quad d$-axis and $q$-axis subtransient time constants, respectively, in $\mathrm{s}$

$V \quad$ Bus terminal voltage magnitude in p.u.

$V_{d}, V_{q} \quad d$-axis and $q$-axis stator voltages, respectively, in p.u.

$V_{g} \quad$ Machine's terminal bus voltage in p.u. and is equal to $V e^{j \theta}$

$V_{r}, V_{\text {ref }}$ AVR-filter voltage and AVR-reference voltage, respectively, in p.u.

$V_{s s} \quad$ AVR-control input (from the PSS or other controller) in p.u.

$X_{d}, X_{q} \quad d$-axis and $q$-axis synchronous reactances, respectively, in p.u.

$X_{d}^{\prime}, X_{q}^{\prime} \quad d$-axis and $q$-axis transient reactances, respectively, in p.u.

$X_{d}^{\prime \prime}, X_{q}^{\prime \prime} \quad d$-axis and $q$-axis subtransient reactances, respectively, in p.u.

$X_{l} \quad$ Armature leakage reactance in p.u.

\section{INTRODUCTION}

$\mathbf{R}$ OTOR angle stability is essential for the stability of power systems. It refers to "the ability of synchronous machines of an interconnected power system to remain in synchronism after being subjected to a disturbance" [1]. A disturbance to the system can be a large one, such as a three-phase fault, or a small one, such as a small step-change in system load. The ability of a power system to recover from a large disturbance is referred to as transient stability, while its ability to adequately damp all the system-oscillations after a small disturbance is referred to as small signal stability.

Corrective measures should be taken in a timely manner after a disturbance, otherwise it can lead to system separation-a phenomenon in which the system divides into two groups of machines and there is a loss of synchronism between the groups - and ultimately to wide scale blackouts and/or islanding in the system. In order to ensure that such a loss in synchronism does not occur, protective and control actions should be taken as soon as a disturbance is detected. For example, a protective action in case of large disturbances is to clear a fault within its critical clearing time. An example of control action for both large and small disturbances is to damp the ensuing system oscillations using power system stabilizers (PSSs) and/or power oscillation dampers (PODs).

Majority of the control actions which are taken after a disturbance in a power system use linear control theory. This requires linearization of power system dynamics at a particular equilibrium point or at a finite set of equilibrium points [2]-[4]. Power systems are nonlinear in nature and a large disturbance (and 139 sometimes even a small one [5]) can alter the operating point of 140 the system quite significantly from equilibrium condition(s) [6]. 141 As linearization is applicable only in the vicinity of equilibrium 142 condition(s), application of linear control methods can prove to 143 be ineffective at the altered condition, and can possibly have an 144 adverse effect on system stability [5], [6]. A logical solution to 145 this problem is to use nonlinear control methods which remain 146 valid for any operating condition and not only for conditions 147 which are close to equilibrium. This solution is the focus of this 148 paper.

The nonlinear control methods which have been proposed in the power system literature can broadly be divided into two categories: methods based on normal forms [7]-[12], and methods based on Lyapunov functions [13]-[17]. In methods based on normal forms, the dynamics of the system are transformed into a new form (called as a normal form) using a curvilinear coordinate system. The transformed system is such that some or all of the transformed states are defined by linear differential equations, and at the same time nonlinearity of the system is exactly preserved in the new form. This transformation process is known as feedback linearization.

In Lyapunov function based methods, a scalar energy-like function of the system states is found (called as Lyapunov function) such that its value is positive at every operating point (except at equilibrium, where it is zero), and the function is non-increasing along any trajectory of the system.

An advantage of normal form based methods over Lyapunov based methods is that a general technique does not exist for finding a Lyapunov function for a system, while the steps for finding a normal form are well established. Moreover, as a normal form is either fully or partially linear, linear control techniques can be used for the linear part of the normal form, whereas linear theory is not applicable in general for Lyapunov based methods. Owing to these advantages, methods based on normal forms have been considered in detail in this paper.

Over the past twenty five years, an in-depth exploration of nonlinear control methods based on normal forms has been conducted for power systems, but almost all of these methods rely on model simplification and approximations [7]-[12]. Specifically, some shortcomings of the existing methods are as follows.

1) Classical model is used in these methods to derive the normal form of power systems. Classical model is a reduced order representation of a synchronous machine, and the transient dynamics of the system are incorrectly reflected in this model. Thus, using a control law which has been derived using this model can have unexpected or unwanted effects on the stability of the system.

2) The final control expression which is obtained in these methods is a function of unmeasurable states of the power system such as rotor angle and transient flux. Approximations are made in order to represent this control expression as a function of measurable quantities, such as stator current and stator terminal voltage. These measurements are acquired from instrument transformers and phasor measurement units (PMUs), and noise, harmonics 
and bad-data are present in them [18]-[22]. Thus, the control expression can become grossly erroneous if it is approximated using these measurements, thereby impacting the stability of the system in a negative manner.

The theory of normal forms has been further explored in this paper in order to address the above shortcomings, and a more robust and practical nonlinear control method has been proposed. The key contributions of this paper are as follows.

1) A detailed subtransient model of machines has been used for developing the control method. This is the recommended model for transient stability analysis as per IEEE Std 1110-2002 [23] (also see [24]) and adequately models transient dynamics in power systems.

2) The aforementioned problem of gross approximation in the final control expression has been eliminated by using estimates of states instead of using directly measured quantities.

3) The proposed method is completely decentralized and only requires local measurements at each generation unit.

4) It has been rigorously shown that the power system remains asymptotically stable under the proposed control.

5) The adverse effects of the aforementioned approximations on the stability of the system have been demonstrated.

Rest of the paper is organized as follows. Section II briefly describes the basics of nonlinear control using normal forms, while Sections III-V develop this theory for power systems. Section VI explains the application of dynamic estimation in the developed nonlinear control. Section VII presents simulations to demonstrate the practical applicability and implementability of the method using a benchmark power system model, and Section VIII concludes the paper.

\section{BASICS OF CONTROL USING NORMAL FORMS}

A general multi-input-multi-output (MIMO) nonlinear system with an equal number of inputs and outputs can be represented in the following form.

$$
\dot{\boldsymbol{x}}=\boldsymbol{f}(\boldsymbol{x})+\sum_{i=1}^{m} \boldsymbol{g}_{i}(\boldsymbol{x}) u_{i} ; y_{i}=h_{i}(\boldsymbol{x}), i=1,2, \ldots, m
$$

It will be shown in Section III that a power system can also be represented as (1). Some preliminary definitions which are required to transform (1) to a normal form are as follows [25].

Definition 1: Lie derivative: The Lie derivative of a differentiable scalar function $h(\boldsymbol{x})$ of vector $\boldsymbol{x}$ along a vector field $\boldsymbol{f}(\boldsymbol{x})$, such that $\boldsymbol{f}$ has same dimension as $\boldsymbol{x}$, is defined as:

$$
L_{\boldsymbol{f}} h(\boldsymbol{x})=\frac{\partial h(\boldsymbol{x})}{\partial \boldsymbol{x}} \boldsymbol{f}(\boldsymbol{x})
$$

Definition 2: Relative degree: For a MIMO nonlinear system given by (1), the relative degree of output $y_{i}$ with respect to the input vector $\boldsymbol{u}$ at a state $\boldsymbol{x}$ is the smallest integer $r_{i}$ such that $L_{\boldsymbol{g}_{j}} L_{\boldsymbol{f}}^{r_{i}-1} h_{i}(\boldsymbol{x}) \neq 0$ for at least one $j \in\{1,2, \ldots, m\}$.

Definition 3: Relative degree set: A MIMO nonlinear system given by (1) has a relative degree set $\left\{r_{1}, r_{2}, \ldots, r_{m}\right\}$ at a state $\boldsymbol{x}$ if $r_{i}$ is the relative degree of $y_{i}, \forall i \in\{1,2, \ldots, m\}$ and the $(m \times m)$ characteristic matrix $\boldsymbol{C}(\boldsymbol{x})$, with $(i, j)$ element as
$C^{i j}(\boldsymbol{x})=L_{\boldsymbol{g}_{j}} L_{\boldsymbol{f}}^{\left(r_{i}-1\right)} h_{i}(\boldsymbol{x})$, is nonsingular. If such a set exists, the system is said to have a well defined relative degree.

With the above three definitions as a base, following results are obtained in order to derive a normal form for a MIMO system described by (1) (as explained in detail in [25]).

Result 1: If a relative degree set $\left\{r_{1}, r_{2}, \ldots, r_{m}\right\}$ exists for a system given by (1), then $r=r_{1}+r_{2}+\cdots+r_{m} \leq n$, where $n$ is the total number of states of the system.

Result 2: (a) Provided that $r \leq n$, where $r=r_{1}+r_{2}+$ $\cdots+r_{m}$ and $r_{i}$ is the relative degree of $i$ th output, define $r$ new states as $z_{j}^{i}=\phi_{j}^{i}(\boldsymbol{x})=L_{\boldsymbol{f}}^{(j-1)} h_{i}(\boldsymbol{x})$ where, for each $i \in\{1,2, \ldots, m\}, j$ is such that $1 \leq j \leq r_{i}$. Define $\boldsymbol{z}^{i}=$ $\left[z_{1}^{i} z_{2}^{i} \ldots z_{r_{i}}^{i}\right]^{T}$ and $\boldsymbol{z}=\left[\boldsymbol{z}^{1^{T}} \boldsymbol{z}^{2^{T}} \ldots \boldsymbol{z}^{m T}\right]^{T}$. The dynamics for each state of the new state vector $z$ are given as follows (for $1 \leq i \leq m$ ), and are known as linearized dynamics of the system.

$$
\begin{aligned}
z_{1}^{i} & =h_{i}(\boldsymbol{x}), \dot{z}_{j}^{i}=L_{\boldsymbol{f}}^{j} h_{i}(\boldsymbol{x})=z_{j+1}^{i}, 1 \leq j \leq r_{i}-1 \\
\dot{z}_{r_{i}}^{i} & =L_{\boldsymbol{f}}^{r_{i}} h_{i}(\boldsymbol{x})+\sum_{j=1}^{m} L_{\boldsymbol{g}_{j}} L_{\boldsymbol{f}}^{\left(r_{i}-1\right)} h_{i}(\boldsymbol{x}) u_{j}=v_{i} \\
\Rightarrow \boldsymbol{v} & =\boldsymbol{D}(\boldsymbol{x})+\boldsymbol{C}(\boldsymbol{x}) \boldsymbol{u} ; \boldsymbol{D}, \boldsymbol{C} \text { are as in nomenclature }
\end{aligned}
$$

(b) If $r<n$, then define another $(n-r)$ new states, $w_{i}=\phi_{i}(\boldsymbol{x}), \quad 261$ where $(r+1) \leq i \leq n$, such that the nonlinear differentiable 262 mapping of the original states to the new states, $\phi(x)=263$ $\left[\phi_{1}^{1}(\boldsymbol{x}) \ldots \phi_{r_{1}}^{1}(\boldsymbol{x}) \ldots \phi_{1}^{m}(\boldsymbol{x}) \ldots \phi_{r_{m}}^{m}(\boldsymbol{x}) \phi_{r+1}(\boldsymbol{x}) \ldots \phi_{n}(\boldsymbol{x})\right]^{T}, \quad 264$ has a corresponding differentiable inverse mapping, $\phi^{-1}$. That 265 is, if $\phi(x)=(\boldsymbol{z}, \boldsymbol{w})$, then $\boldsymbol{x}=\boldsymbol{\phi}^{-1}(\boldsymbol{z}, \boldsymbol{w})$. It is always possible 266 to find such a mapping for a nonlinear system of form (1) with 267 well defined relative degree. Define $\boldsymbol{w}=\left[w_{r+1} w_{r+2} \ldots w_{n}\right]^{T} . \quad 268$ The dynamics for each state of the state vector $\boldsymbol{w}$ can be written 269 as follows (for $(r+1) \leq i \leq n)$ and are known as internal 270 dynamics of the system.

$$
\begin{gathered}
\dot{w}_{i}=\left[L_{\boldsymbol{f}} \phi_{i}(\boldsymbol{x})+\sum_{j=1}^{m} L_{\boldsymbol{g}_{j}} \phi_{i}(\boldsymbol{x}) u_{j}\right]_{\boldsymbol{x}=\phi^{-1}(\boldsymbol{z}, \boldsymbol{w})} \\
\Rightarrow \dot{\boldsymbol{w}}=\boldsymbol{q}(\boldsymbol{z}, \boldsymbol{w})+\boldsymbol{p}(\boldsymbol{z}, \boldsymbol{w}) \boldsymbol{u} ; \boldsymbol{q}, \boldsymbol{p} \text { as in nomenclature }
\end{gathered}
$$

(c) The linearized dynamics and internal dynamics (given by (3) 272 and (4), respectively) represent the system's normal form. 273

A simple interpretation of (3) is that the output $h_{i}(\boldsymbol{x})$ is 274 repeatedly differentiated with respect to time $\left(r_{i}\right.$ times, to be 275 exact) until the input $\boldsymbol{u}$ appears. After this, $h_{i}(\boldsymbol{x})$ and its time 276 derivatives $\frac{d^{j} h_{i}(\boldsymbol{x})}{d t^{j}}=L_{\boldsymbol{f}}^{j} h_{i}(\boldsymbol{x}), 1 \leq j \leq r_{i}-1$ are denoted as 277 a new state vector $\boldsymbol{z}^{i}$ and their dynamics are in a linear form. 278 Thus, this process is known as feedback linearization and the 279 linearized dynamics can be controlled using a linear gain of $\boldsymbol{z}^{i} 280$ as a state feedback for input $v_{i}$. But, in such a feedback, the 281 system's internal dynamics are unobservable and may be unsta- 282 ble. Thus, for the overall stability of the system, it is necessary 283 that besides the linearized dynamics, the internal dynamics also 284 remain stable for a given feedback control. The following result 285 formally states the stability criterion [25].

Result 3: A MIMO system given by (1), with normal form 287 given by (3), (4), is asymptotically stable for a given initial 288 
condition and a given feedback control if the closed loop linearized dynamics are asymptotically stable (that is the closed loop poles are in the left half plane) and the internal dynamics are asymptotically stable. If the closed loop linearized dynamics are asymptotically stable, the asymptotic stability of internal dynamics is equivalent to the asymptotic stability of zero $d y$ namics, which are derived from (4) by representing $\boldsymbol{u}$ in terms of $\boldsymbol{v}$ (using (3), with $\boldsymbol{x}=\phi^{-1}(\boldsymbol{z}, \boldsymbol{w})$ ) and by setting $\boldsymbol{z}$ and $\boldsymbol{v}$ equal to zero. Thus, zero dynamics are given as follows.

$$
\dot{\boldsymbol{w}}=\boldsymbol{q}(\mathbf{0}, \boldsymbol{w})-\boldsymbol{p}(\mathbf{0}, \boldsymbol{w})\left(\boldsymbol{C}\left(\boldsymbol{\phi}^{-1}(\mathbf{0}, \boldsymbol{w})\right)\right)^{-1} \boldsymbol{D}\left(\phi^{-1}(\mathbf{0}, \boldsymbol{w})\right)
$$

\section{Power System Dynamics AND Their Normal Form}

The subtransient model of machines with four rotor coils in each machine, known as Model 2.2 [23], has been used to study power system dynamics (as explained in Section I) and to derive their normal form. Model of a static automatic voltage regulator (AVR) is also included with the model of each machine. Also, all the loads in the system are assumed to be modelled as constant impedance loads. The dynamic equations for this model are given as follows [2]-[4] (where $i$ refers to the system's $i$ th machine, $1 \leq i \leq m$ ).

$$
\begin{aligned}
& \Delta \dot{\delta}^{i}=\left(\omega^{i}-\omega_{0}\right)=\Delta \omega^{i}\left(\text { where, } \Delta \delta^{i}=\delta^{i}-\delta_{0}^{i}\right) \\
& \Delta \dot{\omega}^{i}=\frac{\omega_{0}}{2 H^{i}}\left(T_{m}^{i}-T_{e}^{i}\right)-\frac{D^{i}}{2 H^{i}} \Delta \omega^{i} \\
& \dot{E}_{d}^{\prime i}=\frac{1}{T_{q 0}^{\prime i}}\left[-E_{d}^{\prime i}-\left(X_{q}^{i}-X_{q}^{\prime i}\right)\left[K_{q 1}^{i} I_{q}^{i}+K_{q 2}^{i} \frac{\Psi_{2 q}^{i}+E_{d}^{\prime i}}{X_{q}^{\prime i}-X_{l}^{i}}\right]\right] \\
& \dot{E}_{q}^{\prime i}=\frac{E_{f d}^{i}-E_{q}^{\prime i}+\left(X_{d}^{i}-X_{d}^{\prime i}\right)\left[K_{d 1}^{i} I_{d}^{i}+K_{d 2}^{i} \frac{\Psi_{1 d}^{i}-E_{q}^{\prime i}}{X_{d}^{\prime i}-X_{l}^{i}}\right]}{T_{d 0}^{\prime i}} \\
& \dot{\Psi}_{1 d}^{i}=\frac{1}{T_{d 0}^{\prime \prime i}}\left[E_{q}^{\prime i}+\left(X_{d}^{\prime i}-X_{l}^{i}\right) I_{d}^{i}-\Psi_{1 d}^{i}\right] \\
& \dot{\Psi}_{2 q}^{i}=\frac{1}{T_{q 0}^{\prime \prime i}}\left[-E_{d}^{\prime i}+\left(X_{q}^{\prime i}-X_{l}^{i}\right) I_{q}^{i}-\Psi_{2 q}^{i}\right] \\
& \dot{E}_{d c}^{\prime i}=\frac{1}{T_{c}^{i}}\left[\left(X_{d}^{\prime \prime} i-X_{q}^{\prime \prime} i\right) I_{q}^{i}-E_{d c}^{\prime i}\right] \\
& \dot{V}_{r}^{i}=\frac{1}{T_{r}^{i}}\left[V^{i}-V_{r}^{i}\right], \text { where, } \\
& E_{f d}^{i}=K_{a}^{i}\left[V_{s s}^{i}+V_{r e f}^{i}-V_{r}^{i}\right], E_{f d \min }^{i} \leq E_{f d}^{i} \leq E_{f d \max }^{i} \\
& {\left[\begin{array}{c}
I_{d}^{i} \\
I_{q}^{i}
\end{array}\right]=\left[\begin{array}{cc}
R_{s}^{i} & X_{q}^{\prime \prime} i \\
-X_{d}^{\prime \prime} & R_{s}^{i}
\end{array}\right]^{-1}\left[\begin{array}{l}
E_{d}^{\prime i} K_{q 1}^{i}-\Psi_{2 q}^{i} K_{q 2}^{i}-V_{d}^{i} \\
E_{q}^{\prime i} K_{d 1}^{i}+\Psi_{1 d}^{i} K_{d 2}^{i}-V_{q}^{i}
\end{array}\right]} \\
& T_{e}^{i}=\frac{\omega_{0}}{\omega^{i}} P_{G}^{i}, P_{G}^{i}=V_{d}^{i} I_{d}^{i}+V_{q}^{i} I_{q}^{i}, Q_{G}^{i}=V_{d}^{i} I_{q}^{i}-V_{q}^{i} I_{d}^{i} \\
& V_{d}^{i}=-V^{i} \sin \left(\delta^{i}-\theta^{i}\right), V_{q}^{i}=V^{i} \cos \left(\delta^{i}-\theta^{i}\right) \\
& I_{g}^{i}=\frac{\left[E_{q}^{\prime i} K_{d 1}^{i}+\Psi_{1 d}^{i} K_{d 2}^{i}+j\left\{E_{d}^{\prime i} K_{q 1}^{i}-\Psi_{2 q}^{i} K_{q 2}^{i}-E_{d c}^{i}\right\}\right] e^{j \delta^{i}}}{R_{s}^{i}+j X_{d}^{\prime \prime} i}
\end{aligned}
$$

Using the facts that $R_{s}<<X_{q}^{\prime \prime}, R_{s}<<X_{d}^{\prime \prime}$ and $R_{s}$ is normally taken as 0 p.u. in power system studies ([2], [26]), the above expression for $I_{d}^{i}$ and $I_{q}^{i}$ is simplified as follows.

$$
\begin{aligned}
I_{d}^{i} & =\left(V_{q}^{i}-E_{q}^{i} K_{d 1}^{i}-\Psi_{1 d}^{i} K_{d 2}^{i}\right) / X_{d}^{\prime \prime} i \\
I_{q}^{i} & =\left(E_{d}^{\prime i} K_{q 1}^{i}-\Psi_{2 q}^{i} K_{q 2}^{i}-V_{d}^{i}\right) / X_{q}^{\prime \prime} i
\end{aligned}
$$

The bus voltages, $\left(V^{i}, \theta^{i}\right), 1 \leq i \leq M$ (total number of buses is $M$ ), are given by the following power flow equations.

$$
\begin{aligned}
& P^{i}=\sum_{j=1}^{M} V^{i} V^{j}\left[G^{i j} \cos \left(\theta^{i}-\theta^{j}\right)+B^{i j} \sin \left(\theta^{i}-\theta^{j}\right)\right] \\
& Q^{i}=\sum_{j=1}^{M} V^{i} V^{j}\left[G^{i j} \sin \left(\theta^{i}-\theta^{j}\right)-B^{i j} \cos \left(\theta^{i}-\theta^{j}\right)\right]
\end{aligned}
$$

In order to apply nonlinear control theory to power systems, 313 the differential and algebraic equations (DAEs) of the power 314 system given by (6) and (8) need to be mathematically shown 315 equivalent to the affine ordinary differential equations (ODEs) 316 given by (1). This equivalence can be shown for a well-defined 317 network configuration. Here, a network configuration is con- 318 sidered to be well-defined if the matrix corresponding to the 319 equivalent admittance of the network exists and is non-singular. 320 One example in which the network configuration is not well 321 defined is during a fault in which the line admittance of one 322 or more lines becomes infinite, and hence the line-admittance 323 matrix is undefined and the equivalence fails to hold for the 324 duration of the fault. For a well-defined network configuration 325 one way to show the equivalence of these DAEs and ODEs is 326 by representing each machine as a current source, $I_{g}^{i}$ (defined 327 in (6)), behind a constant admittance, $1 /\left(R_{s}^{i}+j X_{d}^{\prime \prime} i\right)$. It should 328 be noted that $I_{g}^{i}$ is a function of only the states of $i$ th machine 329 and not of any algebraic variables.

The network equations in (8) can be written as follows using basic relation between voltages and current injections.

$$
\boldsymbol{V}=\boldsymbol{Z}_{A} \boldsymbol{I}_{g} ; \boldsymbol{Z}_{A}=\left(\boldsymbol{Y}_{A}\right)^{-1} ; \boldsymbol{Y}_{A}=\boldsymbol{Y}_{N}+\boldsymbol{Y}_{G}+\boldsymbol{Y}_{L}
$$

Here, $\boldsymbol{V}$ is column vector of bus voltages; $\boldsymbol{I}_{g}$ is column 333 vector of current injections, with $i$ th element equal to $I_{g}^{i}$ if $i$ is a 334 machine bus, else it is equal to zero; $\boldsymbol{Y}_{N}$ is network admittance 335 matrix; $\boldsymbol{Y}_{G}$ is diagonal matrix of machine admittances, with 336 $i$ th diagonal element equal to $1 /\left(R_{s}^{i}+j X_{d}^{\prime \prime} i\right)$ if $i$ is a machine 337 bus, else it is equal to zero; similarly, $\boldsymbol{Y}_{L}$ is diagonal matrix of 338 load admittances; and $\boldsymbol{Y}_{A}$ and $\boldsymbol{Z}_{A}$ are augmented matrices of 339 admittance and impedance, respectively. It should be noted that 340 $\boldsymbol{Y}_{L}$ will change with any change in load, hence, $\boldsymbol{Y}_{L}$ is a time 341 varying quantity, unless the loads are constant impedance loads. 342 Thus, modelling loads as constant impedance loads is required to 343 represent power system DAEs as ODEs given by (1). Also, $\boldsymbol{Y}_{G} 344$ and $\boldsymbol{Y}_{N}$ can always be found for a given well-defined network 345 [2]-[3]. Thus, as it is assumed that network configuration is well 346 defined, $\boldsymbol{Y}_{A}$ and $\boldsymbol{Z}_{A}$ exist and are non-singular. 347

The differential equations in (6) can be written as follows. $\quad 348$

$$
\dot{\boldsymbol{x}}^{i}=\boldsymbol{F}^{i}\left(\boldsymbol{x}^{i}, V_{g}^{i}\right)+\sum_{i=1}^{m} \boldsymbol{g}_{i}^{i}\left(\boldsymbol{x}^{i}\right) u_{i}
$$


Where, $\boldsymbol{x}^{i}$ is column vector of the $i$ th machine's states; $V_{g}^{i}$ is the machine's terminal bus voltage and is equal to $V^{i} e^{j \theta^{i}} ; \boldsymbol{F}^{i}$ is the column vector of differential functions in

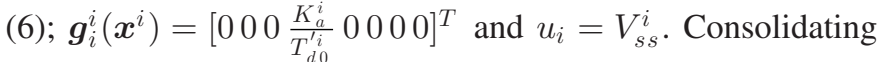
(10) for $i=1,2, \ldots, m$ gives the following equation.

$$
\dot{\boldsymbol{x}}=\boldsymbol{F}\left(\boldsymbol{x}, \boldsymbol{V}_{g}\right)+\sum_{i=1}^{m} \boldsymbol{g}_{i}(\boldsymbol{x}) u_{i}
$$

Here $\boldsymbol{x}=\left[\boldsymbol{x}^{1^{T}} \boldsymbol{x}^{2^{T}} \ldots \boldsymbol{x}^{m T}\right]^{T} ; \boldsymbol{F}=\left[\boldsymbol{F}^{1^{T}} \boldsymbol{F}^{2^{T}} \ldots \boldsymbol{F}^{m T}\right]^{T}$; $\boldsymbol{V}_{g}=\left[V_{g}^{1} V_{g}^{2} \ldots V_{g}^{m}\right]^{T} ; \boldsymbol{g}_{i}(\boldsymbol{x})=\left[\boldsymbol{g}_{i}^{1^{T}} \boldsymbol{g}_{i}^{2^{T}} \ldots \boldsymbol{g}_{i}^{m T}\right]^{T}$, where

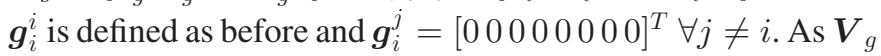
is a subset of $\boldsymbol{V}\left(\boldsymbol{V}_{g}\right.$ only constitutes voltages of machine buses, while $\boldsymbol{V}$ constitutes voltages of all the buses), and $\boldsymbol{V}=\boldsymbol{Z}_{A} \boldsymbol{I}_{g}$ (from (9)), hence $\boldsymbol{V}_{g}$ is also a function of $\boldsymbol{Z}_{A} \boldsymbol{I}_{g}$. Thus, (11) can be written as follows.

$$
\dot{\boldsymbol{x}}=\boldsymbol{F}\left(\boldsymbol{x}, \boldsymbol{Z}_{A} \boldsymbol{I}_{g}\right)+\sum_{i=1}^{m} \boldsymbol{g}_{i}(\boldsymbol{x}) u_{i}
$$

In the above equation, as $\boldsymbol{I}_{g}$ is a function of machine states, $\boldsymbol{x}$, and $\boldsymbol{Z}_{A}$ represents network parameters for a given network configuration, both $\boldsymbol{I}_{g}$ and $\boldsymbol{Z}_{A}$ can be consolidated with $\boldsymbol{F}$ to form a new function $f$ as follows.

$$
\dot{\boldsymbol{x}}=\boldsymbol{f}(\boldsymbol{x})+\sum_{i=1}^{m} \boldsymbol{g}_{i}(\boldsymbol{x}) u_{i}
$$

The above equation is same as (1), and hence this establishes the equivalence of power system DAEs in (6) and (8) and the nonlinear affine ODEs in (1) for a given well-defined network configuration. This idea that DAEs and ODEs are equivalent for a given network configuration has also been used to apply nonlinear control theory to power systems in the current literature [7]-[12]. It should be understood that $f$ changes as soon as there is any change in any line parameter or in network structure, e.g. a change in tap position of a transformer, or if there is any change in load at any bus. Even though $f$ may change, the above equivalence remains valid for the new $f$ as long as $f$ remains well defined, that is the network configuration remains well-defined.

To summarize the above equivalence, with $V_{s s}^{i}$ as input and $\Delta \delta^{i}$ as output, the power system model given by (6)-(8) can be represented as (1), with various terms defined as follows.

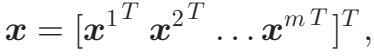

$$
\begin{aligned}
& \boldsymbol{x}^{i}=\left[\Delta \delta^{i} \Delta \omega^{i} E_{d}^{\prime i} E_{q}^{\prime i} \Psi_{1 d}^{i} \Psi_{2 q}^{i} E_{d c}^{\prime i} V_{r}^{i}\right]^{T} ;
\end{aligned}
$$

$\boldsymbol{f}(\boldsymbol{x})$ is obtained from(6)-(8)as described above

$$
\begin{aligned}
& u_{i}=V_{s s}^{i}
\end{aligned}
$$

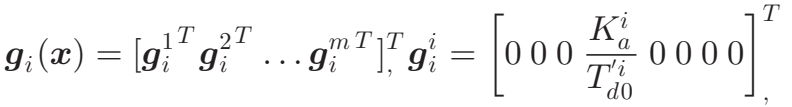

$$
\begin{aligned}
& \boldsymbol{g}_{i}^{j}=\left[\begin{array}{llllllll}
0 & 0 & 0 & 0 & 0 & 0 & 0 & 0
\end{array}\right]^{T} \forall j \neq i ; \\
& y_{i}=h_{i}(\boldsymbol{x})=\Delta \delta^{i}
\end{aligned}
$$

The normal form for the above MIMO representation of 381 power system dynamics is derived as follows.

\section{A. Relative Degree}

The relative degree of output $y_{i}=\Delta \delta^{i}$ for the MIMO system given by (1) and (14) is $r_{i}=3$, as $r_{i}=3$ is the smallest integer for which $L_{\boldsymbol{g}_{j}} L_{\boldsymbol{f}}^{\left(r_{i}-1\right)} \Delta \delta^{i} \neq 0$ for at least some $j \in\{1,2, \ldots, m\}$. That is,

1) $L_{\boldsymbol{g}_{j}} L_{\boldsymbol{f}}^{(1-1)} \Delta \delta^{i}=L_{\boldsymbol{g}_{j}} \Delta \delta^{i}=0 \forall j \in\{1,2, \ldots, m\}$, and

2) $L_{\boldsymbol{g}_{j}} L_{\boldsymbol{f}}^{(2-1)} \Delta \delta^{i}=L_{\boldsymbol{g}_{j}} L_{\boldsymbol{f}} \Delta \delta^{i}=L_{\boldsymbol{g}_{j}} \Delta \omega^{i}=\frac{K_{a}^{j}}{T_{d 0}^{\prime j}} \frac{\partial \Delta \omega^{i}}{\partial E_{q}^{\prime j}}=$ $0 \forall j \in\{1,2, \ldots, m\}$, but

3) $L_{\boldsymbol{g}_{j}} L_{\boldsymbol{f}}^{\left(r_{i}-1\right)} \Delta \delta^{i}=L_{\boldsymbol{g}_{j}} L_{\boldsymbol{f}}^{2} \Delta \delta^{i}=L_{\boldsymbol{g}_{j}} L_{\boldsymbol{f}} L_{\boldsymbol{f}} \Delta \delta^{i}=L_{\boldsymbol{g}_{j}} L_{\boldsymbol{f}}$ $\Delta \omega^{i}=L_{\boldsymbol{g}_{j}}\left[\frac{\omega_{0}}{2 H^{i}}\left(T_{m}^{i}-T_{e}^{i}\right)-\frac{D^{i}}{2 H^{i}} \Delta \omega^{i}\right]=\frac{-\omega_{0} K_{a}^{j}}{2 H^{i} T_{d 0}^{T_{j}^{j}}} \frac{\partial T_{e}^{i}}{\partial E_{q}^{j_{j}}} \neq$ $0 \forall j \in\{1,2, \ldots, m\}$.

The $(i, j)$ element of the $(m \times m)$ characteristic matrix $\boldsymbol{C}(\boldsymbol{x})$ for the system is $C^{i j}(\boldsymbol{x})=L_{\boldsymbol{g}_{j}} L_{\boldsymbol{f}}^{\left(r_{i}-1\right)} \Delta \delta^{i}=\frac{-\omega_{0} K_{a}^{j}}{2 H^{i} T_{d 0}^{T_{j}}} \frac{\partial T_{e}^{i}}{\partial E_{q}^{j}}$. The system has a well defined relative degree provided that $\boldsymbol{C}(\boldsymbol{x})$ is non-singular. But $\boldsymbol{C}(\boldsymbol{x})$ is highly complex and depends not only on the states and parameters of all the machines, but also on various loads and line parameters. Thus, it is very difficult to verify the non-singularity of this matrix. But the existence of a non-singular $\boldsymbol{C}(\boldsymbol{x})$ is not necessary for the existence of a normal form. In case of power systems, the existence of a relative degree for each individual output is sufficient to derive the normal form (as described below).

\section{B. Linearized Dynamics}

As $r_{i}=3$ is the relative degree of $y_{i}=h_{i}(\boldsymbol{x})=\Delta \delta^{i}$, hence $r=r_{1}+r_{2}+\cdots+r_{m}=3 m \leq n=8 m$. According to Result 2(a), $3 m$ new states for the linearized dynamics can be defined, with 3 states for each of the $m$ machines. Using (3), the 3 new states for the $i$ th machine are defined as follows.

$$
\begin{aligned}
& z_{1}^{i}=\Delta \delta^{i}, \dot{z}_{1}^{i}=L_{\boldsymbol{f}} \Delta \delta^{i}=\Delta \dot{\delta}^{i}=\Delta \omega^{i}=z_{2}^{i} \\
& \dot{z}_{2}^{i}=L_{\boldsymbol{f}}^{2} \Delta \delta^{i}=L_{\boldsymbol{f}} \Delta \omega^{i}=\Delta \dot{\omega}^{i}=z_{3}^{i} \\
& \dot{z}_{3}^{i}=L_{\boldsymbol{f}}^{3} \Delta \delta^{i}+\sum_{j=1}^{m} L_{\boldsymbol{g}_{j}} L_{\boldsymbol{f}}^{2} \Delta \delta^{i} u_{j}=\Delta \ddot{\omega}^{i}=v_{i}
\end{aligned}
$$

\section{Internal Dynamics}

The total number of states of the power system are $n=8 m, 412$ and only $3 m$ new states are defined in the linearized dynam- 413 ics. Thus, another $5 m$ states need to be defined for the sys- 414 tem in order to completely represent the system's dynamics, 415 as explained in Result 2(b). One straightforward way to do 416 this is to redefine the states $\left[E_{d}^{\prime i} \Psi_{2 q}^{i} \Psi_{1 d}^{i} E_{d c}^{\prime i} V_{r}^{i}\right]^{T}$ of the $i$ th 417 machine as its new states $\left[w_{1}^{i} w_{2}^{i} w_{3}^{i} w_{4}^{i} w_{5}^{i}\right]^{T}=\boldsymbol{w}^{i}$. It should 418 be noted that the state vector $\boldsymbol{w}=\left[w_{r+1} w_{r+2} \ldots w_{n}\right]^{T}$ in 419 Result 2(b) is same as $\boldsymbol{w}=\left[\boldsymbol{w}^{1^{T}} \boldsymbol{w}^{2^{T}} \ldots \boldsymbol{w}^{m T}\right]^{T}$ in this redefi- 420 nition. This definition is not only simple, but it also has an added 421 advantage that the input $u_{i}=V_{s s}^{i}$ does not affect the dynamic 422 equations of these states directly, but only indirectly through 423 
Therefore, the eight new states for the $i$ th machine, including both the linearized dynamics and the internal dynamics, are given by $\left[z_{1}^{i} z_{2}^{i} z_{3}^{i} w_{1}^{i} w_{2}^{i} w_{3}^{i} w_{4}^{i} w_{5}^{i}\right]^{T}=$ $\left[\Delta \delta^{i} \Delta \omega^{i} \Delta \dot{\omega}^{i} E_{d}^{\prime i} \Psi_{2 q}^{i} \Psi_{1 d}^{i} E_{d c}^{i} V_{r}^{i}\right]^{T}$. Also, equation (16) represents the internal dynamics only when the derivatives $\dot{E}_{d}^{\prime}, \dot{\Psi}_{2 q}^{i}, \dot{\Psi}_{1 d}^{i}, \dot{E}_{d c}^{\prime i}$ and $\dot{V}_{r}^{i}$ are represented in terms of the new states. Substituting the expression for $I_{d}^{i}$ from (7) in the expressions for $\dot{E}_{d}^{\prime i}$ and $\dot{\Psi}_{2 q}^{i}$ from (6), followed by substituting the values of $K_{q 1}^{i}$ and $K_{q 2}^{i}$ (using the nomenclature section), and collecting the coefficients of $E_{d}^{\prime i}, \Psi_{1 d}^{i}$ and $V_{d}^{i}$, the following equations are obtained.

$$
\begin{aligned}
& \dot{E}_{d}^{\prime i}=a_{11}^{i} E_{d}^{\prime i}+a_{12}^{i} \Psi_{2 q}^{i}+\frac{\left(X_{q}^{i}-X_{q}^{\prime i}\right)\left(X_{q}^{\prime \prime} i-X_{l}^{i}\right)}{T_{q 0}^{\prime i} X_{q}^{\prime \prime} i\left(X_{q}^{\prime i}-X_{l}^{i}\right)} V_{d}^{i} \\
& \dot{\Psi}_{2 q}^{i}=a_{21}^{i} E_{d}^{\prime i}+a_{22}^{i} \Psi_{2 q}^{i}+\frac{-\left(X_{q}^{\prime i}-X_{l}^{i}\right)}{T_{q 0}^{\prime \prime i} X_{q}^{\prime \prime} i} V_{d}^{i}, \text { where, } \\
& a_{11}^{i}=\frac{-1}{T_{q 0}^{\prime i}}\left[1+\frac{\left(X_{q}^{i}-X_{q}^{\prime i}\right)\left(X_{l}^{i^{2}}+X_{q}^{\prime i} X_{q}^{\prime \prime} i-2 X_{q}^{\prime \prime} i X_{l}^{i}\right)}{X_{q}^{\prime \prime} i\left(X_{q}^{\prime i}-X_{l}^{i}\right)^{2}}\right] \\
& a_{12}^{i}=\frac{-X_{l}^{i}\left(X_{q}^{i}-X_{q}^{\prime i}\right)\left(X_{q}^{\prime i}-X_{q}^{\prime \prime} i\right)}{T_{q 0}^{\prime i} X_{q}^{\prime \prime} i\left(X_{q}^{\prime i}-X_{l}^{i}\right)^{2}} \\
& a_{21}^{i}=\frac{-X_{l}^{i}}{T_{q 0}^{\prime \prime} i X_{q}^{\prime \prime} i}, a_{22}^{i}=\frac{-X_{q}^{\prime i}}{T_{q 0}^{\prime \prime} i X_{q}^{\prime \prime i}}
\end{aligned}
$$

Similarly, substituting the expressions for $I_{q}^{i}, K_{d 1}^{i}$ and $K_{d 2}^{i}$ in the expression for $\dot{\Psi}_{1 d}^{i}$ from (6), and collecting the coefficients of $E_{d}^{i}, \Psi_{1 d}^{i}$ and $V_{q}^{i}$, the following equation comes.

$$
\dot{\Psi}_{1 d}^{i}=\frac{X_{l}^{i}}{T_{d 0}^{\prime \prime i} X_{d}^{\prime \prime} i} E_{q}^{\prime i}+\frac{-X_{d}^{\prime i}}{T_{d 0}^{\prime \prime} i X_{d}^{\prime \prime} i} \Psi_{1 d}^{i}+\frac{X_{d}^{\prime i}-X_{l}^{i}}{T_{d 0}^{\prime \prime} i X_{d}^{\prime \prime} i} V_{q}^{i}
$$

As $E_{q}^{\prime i}$ is not equal to any of the new states, it should be expressed in terms of the new states in the above equation. Substituting the expressions for $I_{d}^{i}$ and $I_{q}^{i}$ from (7) in the expression for $T_{e}^{i}$ from (6), $T_{e}^{i}$ comes as follows.

$$
T_{e}^{i}=\frac{V_{q}^{i}}{\omega^{i}} \frac{E_{d}^{\prime i} K_{q 1}^{i}-\Psi_{2 q}^{i} K_{q 2}^{i}-V_{d}^{i}}{X_{q}^{\prime \prime} i \omega_{0}}+\frac{V_{d}^{i}}{\omega^{i}} \frac{V_{q}^{i}-E_{q}^{\prime i} K_{d 1}^{i}-\Psi_{1 d}^{i} K_{d 2}^{i}}{X_{d}^{\prime \prime} / \omega_{0}}
$$

Substituting the above expression for $T_{e}^{i}$ in the expression for 446 $\Delta \dot{\omega}^{i}$ from (6), the following equations are derived.

$$
\begin{aligned}
\Delta \dot{\omega}^{i}= & \frac{\omega_{0}}{2 H^{i}} T_{m}^{i}-\frac{\omega_{0}^{2} V_{q}^{i}}{2 H^{i} \omega^{i} X_{q}^{\prime \prime} i}\left(E_{d}^{i i} K_{q 1}^{i}-\Psi_{2 q}^{i} K_{q 2}^{i}-V_{d}^{i}\right) \\
& -\frac{\omega_{0}^{2} V_{d}^{i}}{2 H^{i} \omega^{i} X_{d}^{\prime \prime}}\left(V_{q}^{i}-E_{q}^{i} K_{d 1}^{i}-\Psi_{1 d}^{i} K_{d 2}^{i}\right)-\frac{D^{i}}{2 H^{i}} \Delta \omega^{i} \\
\Rightarrow E_{q}^{\prime} i= & \frac{X_{d}^{\prime \prime} i V_{q}^{i} K_{q 1}^{i}}{X_{q}^{\prime \prime} V_{d}^{i} K_{d 1}^{i}}\left[E_{d}^{i}-\frac{K_{q 2}^{i}}{K_{q 1}^{i}} \Psi_{2 q}^{i}\right]-\frac{K_{d 2}^{i}}{K_{d 1}^{i}} \Psi_{1 d}^{i} \\
& +\frac{X_{q}^{\prime \prime} i-X_{d}^{\prime \prime} i}{X_{q}^{\prime \prime} i} V_{q}^{i} \\
& +\frac{2 H^{i} \omega^{i} X_{d}^{\prime \prime} i}{\omega_{0}^{2} V_{d}^{i} K_{d 1}^{i}}\left[\Delta \dot{\omega}^{i}+\frac{D^{i}}{2 H^{i}} \Delta \omega^{i}-\frac{\omega_{0}}{2 H^{i}} T_{m}^{i}\right]
\end{aligned}
$$

Plugging the above expression for $E_{q}^{\prime i}$ in (18), and collecting 448 the coefficients of $\Psi_{1 d}^{i}$ and $V_{q}^{i}$, the following equation comes. $\quad 449$

$$
\begin{gathered}
\dot{\Psi}_{1 d}^{i}=a_{31}^{i} E_{d}^{i}+a_{32}^{i} \Psi_{2 q}^{i}+a_{33}^{i} \Psi_{1 d}^{i}+\left[\frac{X_{d}^{\prime i}}{X_{d}^{\prime \prime} i}-\frac{X_{l}^{i}}{X_{q}^{\prime \prime} i}\right] \frac{V_{q}^{i}}{T_{d 0}^{\prime \prime i}} \\
+\frac{2 H^{i} \omega^{i} X_{l}^{i}\left(X_{d}^{\prime}-X_{l}\right)}{T_{d 0}^{\prime \prime} \omega_{0}^{2} V_{d}^{i}\left(X_{d}^{\prime \prime}-X_{l}\right)}\left[\Delta \dot{\omega}^{i}+\frac{D^{i}}{2 H^{i}} \Delta \omega^{i}-\frac{\omega_{0}}{2 H^{i}} T_{m}^{i}\right] \\
\text { where, } a_{31}^{i}=\frac{-X_{l}^{\prime i} \tan \left(\delta_{0}^{i}+\Delta \delta^{i}-\theta^{i}\right)\left(X_{q}^{\prime \prime} i-X_{l}^{i}\right)\left(X_{d}^{\prime} i-X_{l}^{i}\right)}{T_{d 0}^{\prime \prime} X_{q}^{\prime \prime}\left(X_{q}^{\prime i}-X_{l}^{i}\right)\left(X_{d}^{\prime \prime}-X_{l}^{i}\right)} \\
a_{32}^{i}=\frac{-\left(X_{q}^{\prime i}-X_{q}^{\prime \prime} i\right)}{\left(X_{q}^{\prime \prime i}-X_{l}^{i}\right)} a_{31}^{i}, a_{33}^{i}=\frac{-\left(X_{d}^{\prime i}-X_{l}^{i}\right)}{T_{d 0}^{\prime \prime} i}
\end{gathered}
$$

Next, the expression for $I_{q}^{i}$ from (7) is substituted in the 450 expression for $\dot{E}_{d c}^{\prime}$ from (6) to obtain the following equation.

$$
\begin{array}{r}
\dot{E}_{d c}^{\prime i}=a_{41}^{i} E_{d}^{\prime i}+a_{42}^{i} \Psi_{2 q}^{i}-\frac{1}{T_{c}^{i}} E_{d c}^{\prime i}-\frac{\left(X_{d}^{\prime \prime} i-X_{q}^{\prime \prime}\right)}{T_{c}^{i} X_{q}^{\prime \prime} i} V_{d}^{i} \\
\text { where, } a_{41}^{i}=\frac{K_{q 1}^{i}\left(X_{d}^{\prime \prime} i-X_{q}^{\prime \prime}\right)}{T_{c}^{i} X_{q}^{\prime \prime} i}, a_{42}^{i}=\frac{-K_{q 2}^{i}\left(X_{d}^{\prime \prime} i-X_{q}^{\prime \prime} i\right)}{T_{c}^{i} X_{q}^{\prime \prime} i}
\end{array}
$$

Finally, using the expressions for $\dot{E}_{d}^{i}, \dot{\Psi}_{2 q}^{i}, \dot{\Psi}_{1 d}^{i}, \dot{E}_{d c}^{i}$ and $\dot{V}_{r}^{i} \quad 452$ (from (17), (20), (21) and (6)) in (16), and replacing $\Delta \delta^{i}, \Delta \omega^{i}, 453$ $\Delta \dot{\omega}^{i}, E_{d}^{\prime i}, \Psi_{2 q}^{i}, \Psi_{1 d}^{i}, E_{d c}^{\prime i}$ and $V_{r}^{i}$ with $z_{1}^{i}, z_{2}^{i}, z_{3}^{i}, w_{1}^{i}, w_{2}^{i}, w_{3}^{i}, \quad 454$ $w_{4}^{i}$ and $w_{5}^{i}$, respectively, the internal dynamics are as follows.

455

$$
\begin{aligned}
& \dot{\boldsymbol{w}}^{i}=\boldsymbol{a}^{i} \boldsymbol{w}^{i}+\boldsymbol{b}^{i}, \forall i \in\{1,2, \ldots, m\} \text { where, } \\
& \boldsymbol{a}^{i}=\left[\begin{array}{ccccc}
a_{11}^{i} & a_{12}^{i} & 0 & 0 & 0 \\
a_{21}^{i} & a_{22}^{i} & 0 & 0 & 0 \\
a_{31}^{i} & a_{32}^{i} & a_{33}^{i} & 0 & 0 \\
a_{41}^{i} & a_{42}^{i} & 0 & \frac{-1}{T_{c}^{i}} & 0 \\
0 & 0 & 0 & 0 & \frac{-1}{T_{r}^{i}}
\end{array}\right], \boldsymbol{b}^{i}=\left[\begin{array}{c}
b_{1}^{i} \\
b_{2}^{i} \\
b_{3}^{i} \\
b_{4}^{i} \\
b_{5}^{i}
\end{array}\right]
\end{aligned}
$$


456

$$
\begin{aligned}
& \boldsymbol{w}^{i}=\left[w_{1}^{i} w_{2}^{i} w_{3}^{i} w_{4}^{i} w_{5}^{i}\right]^{T}, \\
& b_{1}^{i}=\frac{-\left(X_{q}^{i}-X_{q}^{\prime i}\right)\left(X_{q}^{\prime \prime} i-X_{l}^{i}\right)}{T_{q 0}^{\prime i} X_{q}^{\prime \prime} i\left(X_{q}^{\prime i}-X_{l}^{i}\right)} V^{i} \sin \left(\delta_{0}^{i}+z_{1}^{i}-\theta^{i}\right) \\
& b_{2}^{i}=\frac{\left(X_{q}^{\prime i}-X_{l}^{i}\right)}{T_{q 0}^{\prime \prime} X_{q}^{\prime \prime} i} V^{i} \sin \left(\delta_{0}^{i}+z_{1}^{i}-\theta^{i}\right) \text {, } \\
& b_{3}^{i}=\frac{-2 H^{i}\left(\omega_{0}+z_{2}^{i}\right) X_{l}^{i}\left(X_{d}^{\prime}-X_{l}\right)}{T_{d 0}^{\prime \prime} i \omega_{0}^{2} V^{i} \sin \left(\delta_{0}^{i}+z_{1}^{i}-\theta^{i}\right)\left(X_{d}^{\prime \prime}-X_{l}\right)}\left[z_{3}^{i}+\frac{D^{i}}{2 H^{i}} z_{2}^{i}\right. \\
& \left.-\frac{\omega_{0}}{2 H^{i}} T_{m}^{i}\right]+\left[\frac{X_{d}^{\prime i}}{X_{d}^{\prime \prime} i}-\frac{X_{l}^{i}}{X_{q}^{\prime \prime} i}\right] \frac{V^{i} \cos \left(\delta_{0}^{i}+z_{1}^{i}-\theta^{i}\right)}{T_{d 0}^{\prime \prime} i} \\
& b_{4}^{i}=\frac{\left(X_{d}^{\prime \prime}-X_{q}^{\prime \prime} i\right)}{T_{c}^{i} X_{q}^{\prime \prime} i} V^{i} \sin \left(\delta_{0}^{i}+z_{1}^{i}-\theta^{i}\right), b_{5}^{i}=\frac{1}{T_{r}^{i}} V^{i}
\end{aligned}
$$

Elements of $\boldsymbol{a}^{i}$ are as in (17)-(21); $V^{i}, \theta^{i}$ are as in (8) or (9)

Thus, (15) and (22) completely specify a normal form for the power system dynamics.

\section{ASYMPTOTIC StabiLITy OF ZERo DyNAMICS}

The power system will be asymptotically stable under a nonlinear control method based on normal form if both its linearized dynamics and internal dynamics are asymptotically stable, as explained in Section II. The asymptotic stability of linearized dynamics can be ensured using any desired control method (provided that the closed-loop linearized dynamics are not already unstable, or the operating condition has not crossed some threshold of stability, for example, any system fault should be cleared before its critical clearing time (CCT)). From Result 3, if the closed-loop linearized dynamics are stable, then the internal dynamics will be asymptotically stable if and only if the zero dynamics are asymptotically stable.

Lemma 1: The zero dynamics for a power system (with normal form given by (15) and (22)) are asymptotically stable irrespective of operating condition.

Proof: Using Result 3, the zero dynamics of a power system for a given initial operating condition are obtained from its internal dynamics (given by (22)) by putting $z=\mathbf{0}$, that is, by putting $z_{1}^{i}=0, z_{2}^{i}=0$ and $z_{3}^{i}=0$ for all $1 \leq i \leq m$. After substituting $\Delta \dot{\omega}^{i}$ and $\Delta \omega^{i}$ with $z_{3}^{i}$ and $z_{2}^{i}$, respectively, the expression for zero dynamics for $\Delta \dot{\omega}^{i}$ from (6) reduces to $z_{3}^{i}=$ $\frac{\omega_{0}}{2 H^{i}}\left[T_{m}^{i}-T_{e}^{i}\right]-\frac{D^{i}}{2 H^{i}} z_{2}^{i} \Rightarrow T_{m}^{i}-T_{e}^{i}=0\left(\right.$ as $z_{2}^{i}=0$ and $z_{3}^{i}=$ 0 ). Thus, in zero dynamics, $T_{m}^{i}$ and $T_{e}^{i}$ are equal. $T_{m}^{i}$ and $T_{e}^{i}$ can be equal (for all $1 \leq i \leq m$ ) only if the system loads and generations are exactly matched and the line connections and parameters are constant. Thus, $P, Q, B$ and $G$ in (8) remain constant in zero dynamics, and hence $V^{i}$ and $\theta^{i}$ also remain constant for all $1 \leq i \leq M$. The elements of $\boldsymbol{b}^{i}$ in (22) also remain constant for zero dynamics, as they are functions of $z_{1}^{i}$, $z_{2}^{i}, z_{3}^{i}, V^{i}$ and $\theta^{i}$. As $\boldsymbol{b}^{i}$ is a constant for all $1 \leq i \leq m$, the zero dynamics will be asymptotically stable if and only if all the eigenvalues of $\boldsymbol{a}^{i}$ are negative for all $1 \leq i \leq m$ [27]. The eigenvalues of $\boldsymbol{a}^{i}$ are the roots of its following characteristic polynomial.

$$
\begin{aligned}
& \text { determinant }\left(\boldsymbol{a}^{i}-\lambda \boldsymbol{I}\right)=0 \\
& \Rightarrow\left[\left[a_{11}^{i}-\lambda\right]\left[a_{22}^{i}-\lambda\right]-a_{12}^{i} a_{21}^{i}\right]\left[a_{33}^{i}-\lambda\right]\left[\frac{-1}{T_{c}^{i}}-\lambda\right] \\
& \quad \times\left[\frac{-1}{T_{r}^{i}}-\lambda\right]=0
\end{aligned}
$$

Three roots of the above equation are $\lambda=a_{33}^{i}, \lambda=\frac{-1}{T_{c}^{i}}, \lambda=495$ $\frac{-1}{T^{i}}$, and the other two roots are the solutions of the following 496 equation.

$$
\begin{aligned}
& \lambda^{2}+b \lambda+c=0 ; b=-a_{11}^{i}-a_{22}^{i}, c=a_{11}^{i} a_{22}^{i}-a_{12}^{i} a_{21}^{i} \\
& \Rightarrow \lambda=\left(-b \pm \sqrt{b^{2}-4 c}\right) / 2=-\left(b \mp \sqrt{b^{2}-4 c}\right) / 2
\end{aligned}
$$

Using (17), $c$ in the above equation is evaluated as follows.

$$
\begin{aligned}
& c=\frac{-1}{T_{q 0}^{\prime i}}\left[1+\frac{\left(X_{q}^{i}-X_{q}^{\prime i}\right)\left(X_{l}^{i^{2}}+X_{q}^{\prime i} X_{q}^{\prime \prime} i-2 X_{q}^{\prime \prime} i X_{l}^{i}\right)}{X_{q}^{\prime \prime} i\left(X_{q}^{\prime i}-X_{l}^{i}\right)^{2}}\right] \frac{-X_{q}^{\prime i}}{T_{q 0}^{\prime \prime} X_{q}^{\prime \prime} i} \\
& -\frac{-X_{l}^{i}\left(X_{q}^{i}-X_{q}^{\prime i}\right)\left(X_{q}^{\prime i}-X_{q}^{\prime \prime}\right)}{T_{q 0}^{\prime i} X_{q}^{\prime \prime}\left(X_{q}^{\prime i}-X_{l}^{i}\right)^{2}} \frac{-X_{l}^{i}}{T_{q 0}^{\prime \prime} i X_{q}^{\prime \prime i}}=\frac{X_{q}^{\prime i}}{T_{q 0}^{\prime i} T_{q 0}^{\prime \prime} X_{q}^{\prime \prime i}} \\
& +\frac{\left(X_{q}^{i}-X_{q}^{\prime i}\right)\left(X_{q}^{\prime i}\left(X_{l}^{i^{2}}-2 X_{q}^{\prime \prime} i X_{l}^{i}+X_{q}^{\prime i} X_{q}^{\prime \prime} i\right)-X_{l}^{i^{2}}\left(X_{q}^{\prime i}-X_{q}^{\prime \prime} i\right)\right)}{T_{q 0}^{\prime i} T_{q 0}^{\prime \prime} X_{q}^{\prime \prime} i^{2}\left(X_{q}^{\prime i}-X_{l}^{i}\right)^{2}} \\
& =\frac{X_{q}^{\prime i}}{T_{q 0}^{\prime i} T_{q 0}^{\prime \prime i} X_{q}^{\prime \prime i}}+\frac{\left(X_{q}^{i}-X_{q}^{\prime i}\right)\left(X_{q}^{\prime}{ }^{2} X_{q}^{\prime \prime} i i+X_{l}^{i} X_{q}^{\prime \prime} i-2 X_{q}^{\prime i} X_{l}^{i} X_{q}^{\prime \prime} i\right)}{T_{q 0}^{\prime i} T_{q 0}^{\prime \prime} i X_{q}^{\prime \prime} i^{2}\left(X_{q}^{\prime i}-X_{l}^{i}\right)^{2}} \\
& =\frac{X_{q}^{\prime i}}{T_{q 0}^{\prime i} T_{q 0}^{\prime \prime i} X_{q}^{\prime \prime i}}+\frac{\left(X_{q}^{i}-X_{q}^{\prime i}\right)\left(X_{q}^{\prime i}-X_{l}^{i}\right)^{2} X_{q}^{\prime \prime} i}{T_{q 0}^{\prime i} T_{q 0}^{\prime \prime i} X_{q}^{\prime \prime}{ }^{2}\left(X_{q}^{\prime i}-X_{l}^{i}\right)^{2}} \\
& =\frac{X_{q}^{\prime i}}{T_{q 0}^{\prime i} T_{q 0}^{\prime \prime i} X_{q}^{\prime \prime} i}+\frac{X_{q}^{i}-X_{q}^{\prime i}}{T_{q 0}^{\prime i} T_{q 0}^{\prime \prime i} X_{q}^{\prime \prime i}}=\frac{X_{q}^{i}}{T_{q 0}^{\prime i} T_{q 0}^{\prime \prime i} X_{q}^{\prime \prime i}}>0
\end{aligned}
$$

Also, as explained in [26], the following inequalities hold.

$$
X_{q}^{i}>X_{q}^{\prime i} \geq X_{d}^{\prime i}>X_{q}^{\prime \prime} i>X_{l}^{i}>0
$$

Hence, in (17), $a_{11}^{i}<0, a_{12}^{i}<0, a_{21}^{i}<0$ and $a_{22}^{i}<0, \quad 500$ and hence, $b=-a_{11}^{i}-a_{22}^{i}>0$ and $a_{12}^{i} a_{21}^{i}>0$. This also 501 implies that $b^{2}-4 c=\left(a_{11}^{i}+a_{22}^{i}\right)^{2}-4\left(a_{11}^{i} a_{22}^{i}-a_{12}^{i} a_{21}^{i}\right)=502$ $\left(a_{11}^{i}-a_{22}^{i}\right)^{2}+4\left(a_{12}^{i} a_{21}^{i}\right)>0$ and, thus $\sqrt{b^{2}-4 c}$ is a real 503 number and, hence, is positive, and following inequalities exist. 504

$$
\begin{aligned}
& c>0, b>0, \sqrt{b^{2}-4 c}>0 \\
\Rightarrow & b^{2}>b^{2}-4 c \Rightarrow b>\sqrt{b^{2}-4 c}>0 \\
\Rightarrow & \left(b \mp \sqrt{b^{2}-4 c}\right) / 2>0 \Rightarrow-\left(b \mp \sqrt{b^{2}-4 c}\right) / 2<0
\end{aligned}
$$

From (24) and (26), $\lambda=-\left(b \mp \sqrt{b^{2}-4 c}\right) / 2<0$ are two roots of (23) and the other roots are $\lambda=a_{33}^{i}=\frac{-\left(X_{d}^{i_{i}}-X_{l}^{i}\right)}{T_{d 0}^{I_{i}}}<0$ (using (20) and (25)), $\lambda=\frac{-1}{T_{c}^{i}}<0$ and $\lambda=\frac{-1}{T_{r}^{i}}<0$. Thus all the five roots of (23) are negative, and $\boldsymbol{a}^{i}$ has negative eigenvalues for all $1 \leq i \leq m . \therefore$ the zero dynamics are asymptotically stable. 
Also, $\dot{E}_{q}^{i}$ from (7) can be written as follows.

$$
\begin{aligned}
\dot{E}_{q}^{\prime i} & =\frac{1}{T_{d 0}^{\prime i}}\left[E_{f d}^{i}+E_{q}^{i^{\prime \prime}}\right], \text { where } \\
E_{q}^{i \prime \prime} & =\left(X_{d}^{i}-X_{d}^{\prime i}\right)\left[K_{d 1}^{i} I_{d}^{i}+K_{d 2}^{i} \frac{\Psi_{1 d}^{i}-E_{q}^{\prime i}}{X_{d}^{\prime i}-X_{l}^{i}}\right]-E_{q}^{\prime i}
\end{aligned}
$$

Finally, using (6),(28)-(30), the optimal $V_{s s}^{i}$ comes as follows.

$$
\begin{aligned}
V_{s s}^{i}= & \frac{E_{f d}^{i}}{K_{a}^{i}}-V_{r e f}^{i}+V_{r}^{i}, E_{f d \min }^{i} \leq E_{f d}^{i} \leq E_{f d \max }^{i} \\
E_{f d}^{i}= & \frac{T_{e}^{i^{\prime}}-\left[7.07 \Delta \delta^{i}+13.65 \Delta \omega^{i}+\left[6.11-\frac{D^{i}}{2 H^{i}}\right] \Delta \dot{\omega}^{i}\right] \frac{2 H^{i}}{\omega_{0}}}{\left(\omega_{0} V_{d}^{i} K_{d 1}^{i}\right) /\left(\omega^{i} X_{d}^{\prime \prime} T_{d 0}^{\prime i}\right)} \\
& -E_{q}^{i^{\prime \prime}} \\
& \text { where, } T_{e}^{i^{\prime}}, E_{q}^{i^{\prime \prime}} \text { are defined in }(29),(30), \text { resp. }
\end{aligned}
$$

Remark 1: Although the normal form for power system dynamics, their asymptotic stability and the final control expression have been derived using a static AVR, these can be similarly derived considering other types of AVRs as well.

Remark 2: The control input $V_{s s}^{i}$ in (31) is a small-signal input given to a machine's AVR. It should be zero in steadystate, and should be non-zero only for around $10 s-20 s$ after a disturbance (which is the time-frame of interest for rotor angle stability). Thus, $V_{s s}^{i}$ should be passed through a washout filter to filter-out any DC-component in it and then be given as an input to the AVR. The largest time constant which describes the dynamics corresponding to rotor angle stability is given by $T_{d 0}^{\prime}$, which is around 3-10 s for a thermal unit in a power system [26]. $T_{d 0}^{\prime}$ for the machines considered in Section VII is around $5 \mathrm{~s}$. Also, the time period of any oscillatory mode which is of interest (including inter-area modes) is not more than $5 \mathrm{~s}$. Thus, time constant of the washout filter is taken as 5 s, so that any slow dynamics which have time constant higher than $5 \mathrm{~s}$ (or any DC components) are filtered out.

As the washout filter removes any DC components in the signal, and as $\Delta \dot{\omega}^{i}=\left(\dot{\omega}^{i}-\dot{\omega}_{0}^{i}\right), \Delta \omega^{i}=\left(\omega^{i}-\omega_{0}^{i}\right)$ and $\Delta \delta^{i}=$ $\left(\delta^{i}-\delta_{0}^{i}\right)$ are DC signals in the post-fault equilibrium, when the fault is removed and system stabilizes to a new steady state (and hence to a new topology), these DC components are also filtered-out by the washout filter. Thus post-fault rotor angle and rotor velocity can be different from their pre-fault values, and the post-fault dynamics can easily adjust to any topological changes in the system. Also, due to filtering-out of the DC-components by the washout filter, any initial values of $\dot{\omega}_{0}^{i}, \omega_{0}^{i}$ and $\delta_{0}^{i}$ can be taken, and in this paper these are taken as 0 p.u., 1 p.u. and $0 \mathrm{rad}$, respectively.

Remark 3: The stability criteria given in Sections IV and V will remain valid even if saturation of the unit is considered. This is because saturation in synchronous machines is manifested as changes in its parameters (see [23]), that is, changes in $X_{d}^{i}, X_{q}^{i}$, $X_{d}^{\prime}, X_{q}^{\prime}$, and so on. Thus, even if these parameters get altered because of saturation, the relation given by (25) still remains valid, and hence the stability criteria given in Sections IV and $\mathrm{V}$ are valid.

\section{DECENTRALIZED NONLINEAR ESTIMATION}

The control expression in (31) is a function of unmeasurable system states, and hence these states must be estimated in realtime to implement the control. With the introduction of PMUs in power systems, it is now possible to acquire in real-time various phasor quantities, such as bus voltages and line currents, at very 
high sampling frequencies $(>100 \mathrm{~Hz})[20]-[22]$. These phasor measurements can be utilized for estimation of the unmeasurable states and this estimation is known as dynamic state estimation (DSE) [28]-[33]. In [31] a method was proposed to perform DSE in a decentralized and nonlinear manner. In this method, the voltage phasor $\left(V^{i}, \theta^{i}\right)$ acquired at $i$ th machine's terminal bus is treated as an input in order to decouple the dynamic equations of a machine from rest of the power system, followed by application of unscented Kalman filtering (UKF, [34])-a nonlinear method of DSE.

UKF is a discrete and iterative method that has four steps. First, a set of points, called as sigma points, is generated at each time sample such that the mean and covariance of the points, respectively, is equal to the estimated mean and covariance of the state estimates from the previous time sample. In the second step, the sigma points are passed through the dynamic state equations (in this case, the decoupled dynamic equations of the machine) to get the predicted sigma points and predicted state estimates. In the third step, the predicted sigma points are used to generate predicted measurements, and finally, the predicted state estimates and the predicted measurements are corrected using the actual measurement(s) (in this case, the actual measurement is current phasor measured at machine terminal) and the states estimates are thus generated.

Using the above method, the estimates of $\Delta \delta^{i}, \Delta \omega^{i}, E_{q}^{\prime i}$, $E_{d}^{i}, \Psi_{2 q}^{i}, \Psi_{1 d}^{i}, V_{r}^{i}$ are found for the $i$ th machine using just the voltage and current phasors measured at that machine. These estimates are then used in (31) to find the input $V_{s s}^{i}$ for the machine's AVR at each time sample. The estimate of derivative of a term used in (31) is found by subtracting the estimate of the term in the previous sample from its estimate in the current sample, and dividing the difference by the sampling period. For example, estimate of $\dot{V}_{q}^{i}(k)$ is $\frac{V_{q}^{i}(k)-V_{q}^{i}(k-1)}{T_{0}}$, where $k$ denotes the $k$ th time sample, $T_{0}$ is the sampling period, and $V_{q}^{i}(k)=V^{i}(k) \cos \left(\delta_{0}^{i}+\Delta \delta^{i}(k)-\theta^{i}(k)\right)$ (from (6)).

Remark 4: As $V_{d}^{i}$ and $V_{q}^{i}$ are algebraic quantities, their timederivatives can have very large magnitudes during switching events or any other disturbances, and hence these large magnitude derivatives should be detected and filtered-out as they can jeopardize the stability of the system. In this paper, this is done by comparing the derivative at the $k$ th time sample with the derivative at $(k-1)$ th time sample, and if the difference between the two is more than a predetermined value, then the derivative at the $k$ th time sample is set to zero. In this paper, this predetermined value is taken as 0.04 , as this value successfully removes all such large magnitude derivatives, and at the same time retains other derivatives. This value was found using trial and error.

A model 16-machine, 68-bus test system (Fig. 1) has been used for the case study and MATLAB-Simulink has been used for its modelling and simulation. A detailed description of the system is available in [4] or [35]. The following two cases have been considered to assess the performance of the proposed control method for both small signal stability and transient stability.

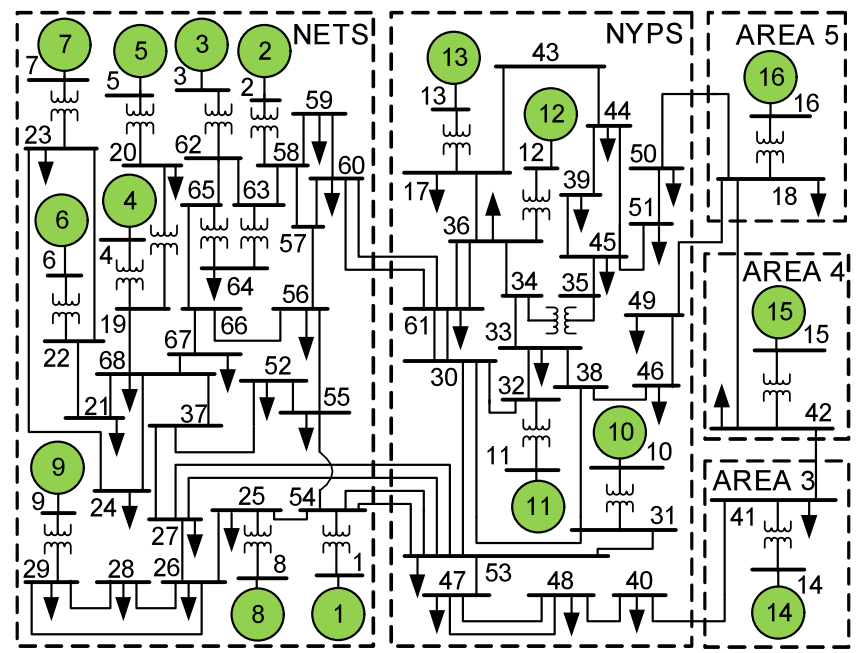

Fig. 1. Line diagram of the 16-machine, 68-bus, power system model.

TABLE I

MOdAL ANALYSIS OF INTERAREA MODES

\begin{tabular}{lcccc}
\hline \hline & $\begin{array}{c}\text { Open- } \\
\text { loop }\end{array}$ & $\begin{array}{c}\text { PSS } \\
\text { control }\end{array}$ & $\begin{array}{c}\text { ELQR } \\
\text { control }\end{array}$ & $\begin{array}{c}\text { Nonlinear } \\
\text { control }\end{array}$ \\
\hline Mode-1 frequency (Hz) & 0.39 & 0.43 & 0.31 & 0.12 \\
Mode-1 damping ratio (\%) & 0.9 & 11.4 & 18.7 & 47.2 \\
\hline Mode-2 frequency (Hz) & 0.52 & 0.54 & 0.47 & 0.20 \\
Mode-2 damping ratio (\%) & 2.1 & 6.3 & 9.8 & 92.8 \\
\hline Mode-3 frequency (Hz) & 0.60 & 0.63 & 0.54 & 0.21 \\
Mode-3 damping ratio (\%) & 1.2 & 5.7 & 11.0 & 91.0 \\
\hline \hline
\end{tabular}

Case A. Assessment of Small Signal Stability: In this case, the test system starts from steady state, and at $t=1 \mathrm{~s}$ a smalldisturbance takes place in which one of the tie-lines of the double circuit line between buses 54-53 goes out of service. The test system has four inter-area modes in the range 0.1$1.0 \mathrm{~Hz}$ and three of these modes are very poorly damped with damping ratios less than $3 \%$ (Table I). Thus, after the smalldisturbance, poorly damped oscillations ensue in the open-loop system, which need to be controlled using an adequate method of control. Three different control methods have been considered to control the oscillations: (1) using PSS control, (2) using decentralized linear control, and (3) using the proposed non-linear control. The employed decentralized linear control is based on an extended linear quadratic regulator (ELQR), and has been described in [36]-[37]. A description of the PSS control method and its parameters is also available in [37].

The plots of the power flow in the inter-area line between buses 60-61 for the three control types are shown in Fig. 2 and the plots for $\delta, \omega, V$ and $E_{f d}$ of units 1 and 8 are shown in Figs. 3 and 4. Units 1 and 8 have been chosen for showing the plots because the disturbance takes close to these units. Also, the plots for a unit which is not close to the disturbance (unit 13) are shown in Fig. 5. It can be observed in Figs. 2-5 that the damping provided to the oscillations by the proposed control is better than the other two controls. Also, the voltage regulation provided by the proposed control is on a par with the other two methods. 


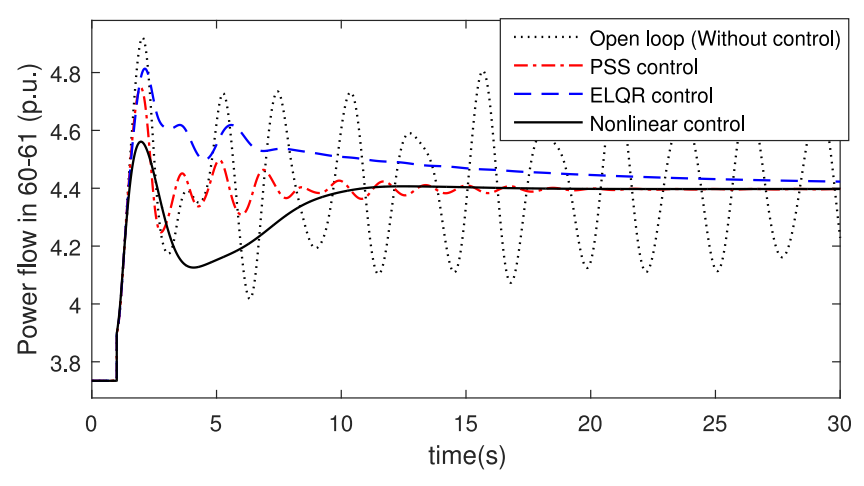

Fig. 2. Small-signal stability comparison: Power flow in interarea line.
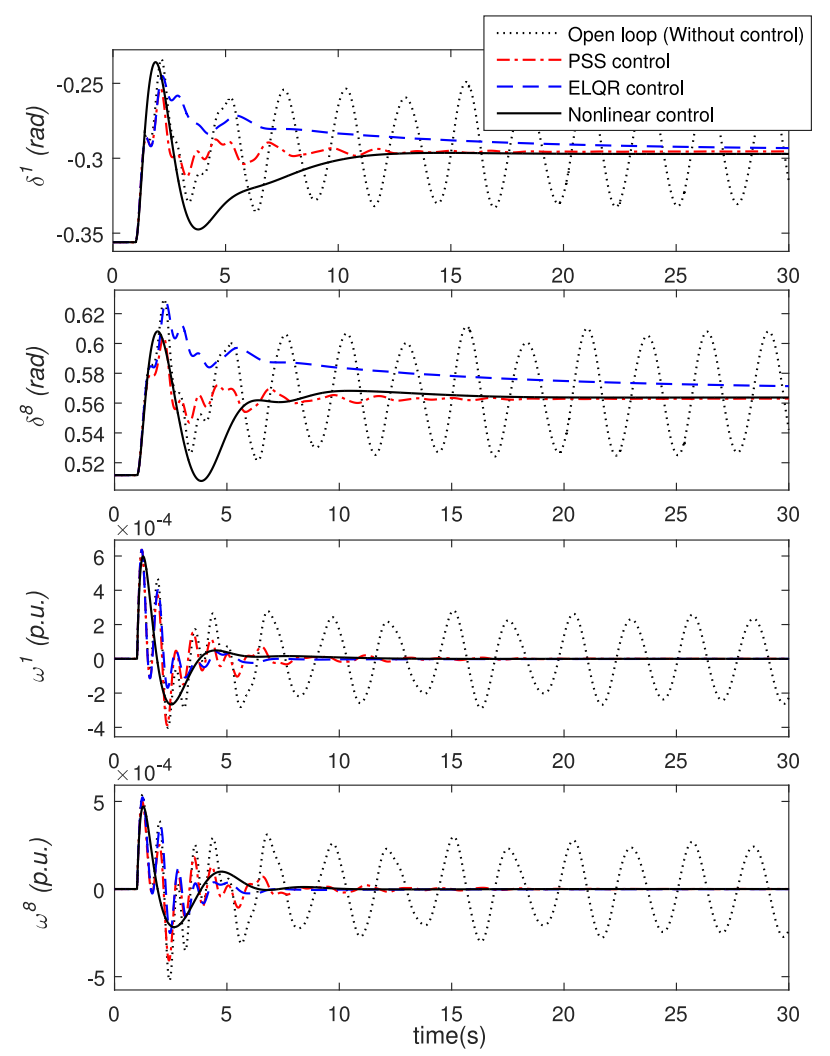

Fig. 3. Small-signal stability comparison: $\delta$ and $\omega$ for units 1 and 8 .
Modal and sensitivity analysis: Table I presents the modal analysis of interarea modes, wherein the frequencies and damping ratios of the three poorly damped inter-area modes have been shown. It can be seen in Table I that the damping ratios for the proposed control are much higher as compared to the other two control methods, while the corresponding frequencies for the proposed control are much lower. To understand the reason behind this, further analysis of the sensitivity of the interarea modes to system states needs to be done.

The sensitivity of a system's $a$ th mode (or eigenvalue) to the system's $b$ th state is given by the participation factor $P_{b a}$, and is equal to the product of the $b$ th entry of the $a$ th right eigenvector and the $b$ th entry of the $a$ th left eigenvector [26]. As the interarea modes are the most significant electromechanical modes of the test system, it is logical to see how sensitive these modes are to the various electromechanical states of the system
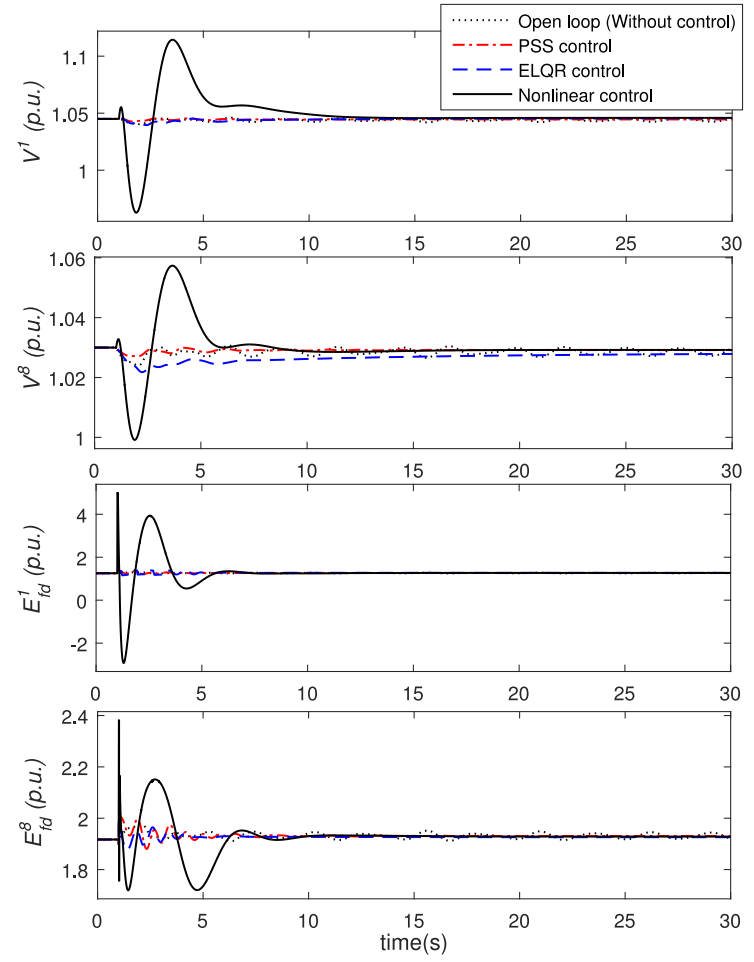

Fig. 4. Small-signal stability comparison: $V$ and $E_{f d}$ for units 1 and 8.
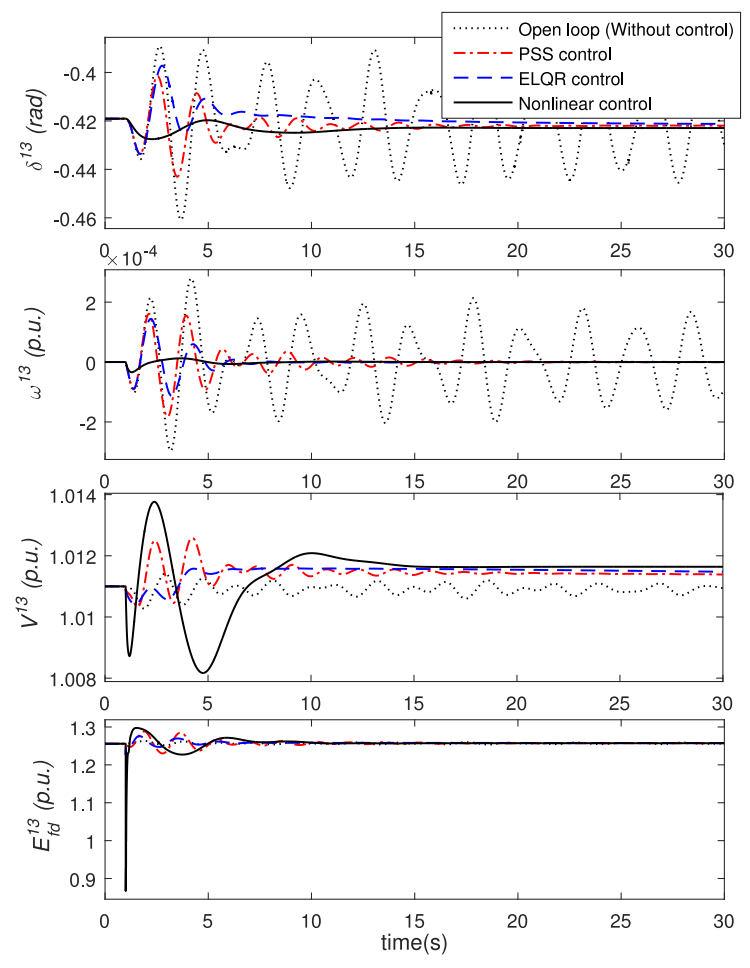

Fig. 5. Small-signal stability comparison for unit 13.

(electromechanical states are the $\delta$ and $\omega$ of various generating 682 units in the system). Table II shows the top three normalized 683 participation factors (NPF) of the electromechanical states in 684 each of the interarea modes for the four control scenarios.

It can be seen that the participation in the three interarea 686 modes from various electromechanical states is among the 687 
TABLE II

SENSITIVITY ANALYSIS: NORMALIZED PARTICIPATION FACTORS (NPF) OF ELECTROMECHANICAL STATES IN THE THREE INTERAREA MODES

\begin{tabular}{lcccccc}
\hline \hline & \multicolumn{2}{c}{ Mode-1 } & \multicolumn{2}{c}{ Mode-2 } & \multicolumn{2}{c}{ Mode-3 } \\
\hline \multirow{4}{*}{ Open-loop } & State & NPF & State & NPF & State & NPF \\
& $\delta^{15}$ & 1.00 & $\omega^{16}$ & 1.00 & $\omega^{13}$ & 1.00 \\
& $\omega^{15}$ & 0.99 & $\delta^{16}$ & 0.99 & $\delta^{13}$ & 0.99 \\
& $\delta^{14}$ & 0.84 & $\delta^{14}$ & 0.81 & $\omega^{16}$ & 0.20 \\
\hline \multirow{3}{*}{ PSS control } & $\omega^{13}$ & 1.00 & $\omega^{16}$ & 1.00 & $\omega^{13}$ & 1.00 \\
& $\omega^{15}$ & 0.89 & $\delta^{16}$ & 0.93 & $\delta^{13}$ & 0.92 \\
& $\delta^{15}$ & 0.83 & $\omega^{14}$ & 0.73 & $\omega^{16}$ & 0.13 \\
\hline \multirow{3}{*}{ ELQR control } & $\delta^{1}$ & 1.00 & $\delta^{16}$ & 1.00 & $\omega^{13}$ & 1.00 \\
& $\omega^{15}$ & 0.96 & $\omega^{16}$ & 0.97 & $\delta^{13}$ & 0.98 \\
& $\delta^{15}$ & 0.94 & $\omega^{14}$ & 0.94 & $\delta^{16}$ & 0.32 \\
Nonlinear control & $\delta^{14}$ & 0.30 & $\omega^{15}$ & 0.25 & $\omega^{13}$ & 0.39 \\
& $\delta^{15}$ & 0.27 & $\omega^{14}$ & 0.10 & $\omega^{12}$ & 0.10 \\
& $\delta^{16}$ & 0.26 & $\omega^{16}$ & 0.07 & $\omega^{1}$ & 0.10 \\
\hline \hline
\end{tabular}

TABLE III

TOP THREE NoRMALIZEd PARTICIPATION FACTORS (NPF) OF SySTEM STATES IN THE INTERAREA MODES FOR THE PROPOSED CONTROL

\begin{tabular}{ccccccc}
\hline \hline & \multicolumn{2}{c}{ Mode-1 } & \multicolumn{2}{c}{ Mode-2 } & \multicolumn{2}{c}{ Mode-3 } \\
\hline & State & NPF & State & NPF & State & NPF \\
\hline \multirow{4}{*}{ Nonlinear control } & $\hat{\dot{V}}_{q}^{16}$ & \multirow{2}{*}{1.00} & $\hat{\dot{V}}_{q}^{15}$ & \multirow{2}{*}{1.00} & $\hat{\dot{V}}_{q}^{13}$ & \multirow{2}{*}{1.00} \\
& $\hat{\dot{V}}_{d}^{16}$ & 0.75 & $\hat{\Psi}_{1 d}^{15}$ & 0.61 & $\hat{\omega}^{13}$ & 0.80 \\
& $\hat{\Psi}_{1 d}^{16}$ & 0.63 & $\hat{E}_{q}^{\prime 15}$ & 0.52 & $\hat{\Psi}_{1 d}^{13}$ & 0.76 \\
\hline \hline
\end{tabular}

highest for three scenarios: open-loop, PSS control and ELQR control. But this participation is significantly reduced for the proposed nonlinear control. Instead, the highest participating states in the case of nonlinear control are various controller states, as can be seen in Table III (the hat, ${ }^{\wedge}$, denotes that the quantities are estimated controller states). This high participation of controller states in the interarea modes is the reason behind relatively low frequencies, and relatively higher damping ratios for the interarea modes.

The overall system damping is decided not only by the frequencies of the dominant modes, but also by the damping ratios of those modes [26]. Thus, the reduction in frequencies of the interarea modes in the case of nonlinear control has no adverse effect on control performance because this reduction is well compensated by a significant increase in damping ratios, and the overall damping provided by the proposed control is better than the other two control methods.

Remark 5. Performance of other methods of nonlinear control: Other methods of nonlinear control have also been tested for controlling the oscillations caused by the aforementioned small disturbance in the test system. These methods include control using two methods based on normal forms given in [7] and [8], and control using two Lyapunov based methods given in [13] and [16]. It was found that the methods either destabilize the system or produce unwanted oscillations (power flow in line 60-61 for the methods based on normal forms are shown in Fig. 6 ). This is because all these tested methods have been derived using classical model of machines and employ gross approximations, and hence have adverse effects on the stability of

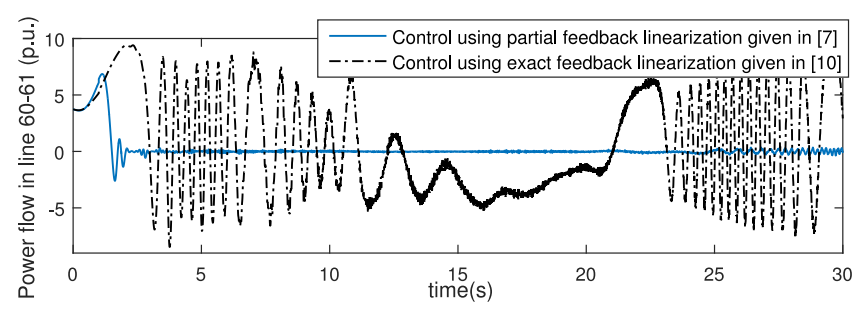

Fig. 6. Performance of other methods of control based on normal forms.

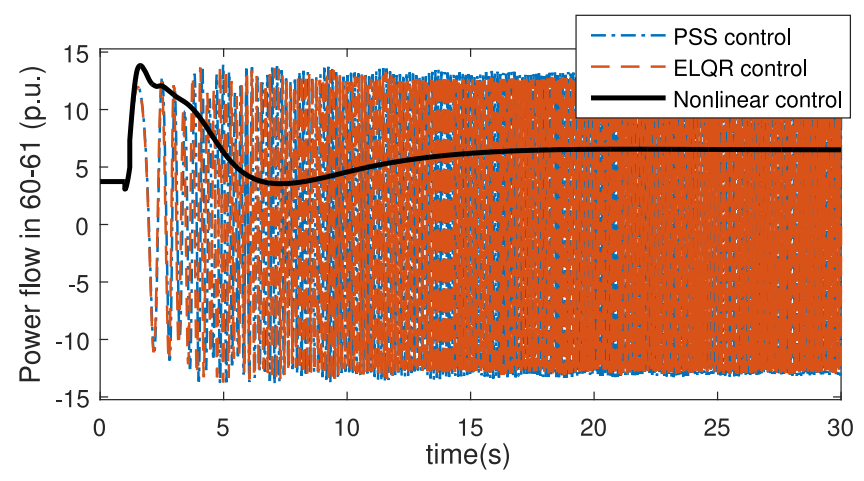

Fig. 7. Transient stability comparison: Power flow in interarea line.

the test system which is modelled using a detailed subtransient 717 model (IEEE Model 2.2) for machines.

Case B. Assessment of Transient Stability In the second case, the system starts from steady state, and at $t=1 \mathrm{~s}$ a three phase fault is simulated at bus 54 , followed by clearing the fault after $200 \mathrm{~ms}$ by opening of circuit breakers on the line 54-53. Fig. 7 shows the response of the three control methods to this large disturbance. Other nonlinear methods of control have not been used for comparison as they failed to perform properly even for a small disturbance (Remark 5). It can be clearly observed that the proposed method can provide adequate damping to the oscillations occurring after the disturbance, and hence can ensure the transient stability of the system after a large-disturbance. On the other hand, the system destabilizes after the disturbance under either PSS control or ELQR control. As both PSS and ELQR fail to stabilize the system, in Figs. 8-10 only the performance under the proposed nonlinear has been shown.

Discussion on magnitude of control input and the control performance: The final control input which is given to a generator is the excitation voltage, $E_{f d}$, from the corresponding AVR. It can be observed in Figs. 4, 5, 9, and 10 that the control input is larger for nonlinear control as compared to PSS or ELQR. A relatively larger control input in case of nonlinear control is needed in order to ensure that the control can maintain the stability of the system in face of both large and small disturbances. This observation of nonlinear control requiring relatively larger control input has been made in power system literature, such as [7], [8]. It can also be observed that nonlinear control provides a large control input to the generators only for the first four-five seconds after the disturbance, and within around 15 seconds the control inputs, the states and other algebraic variables of generators settle down to their steady-state values. Also, the control input is larger for generators which are electrically close to the 

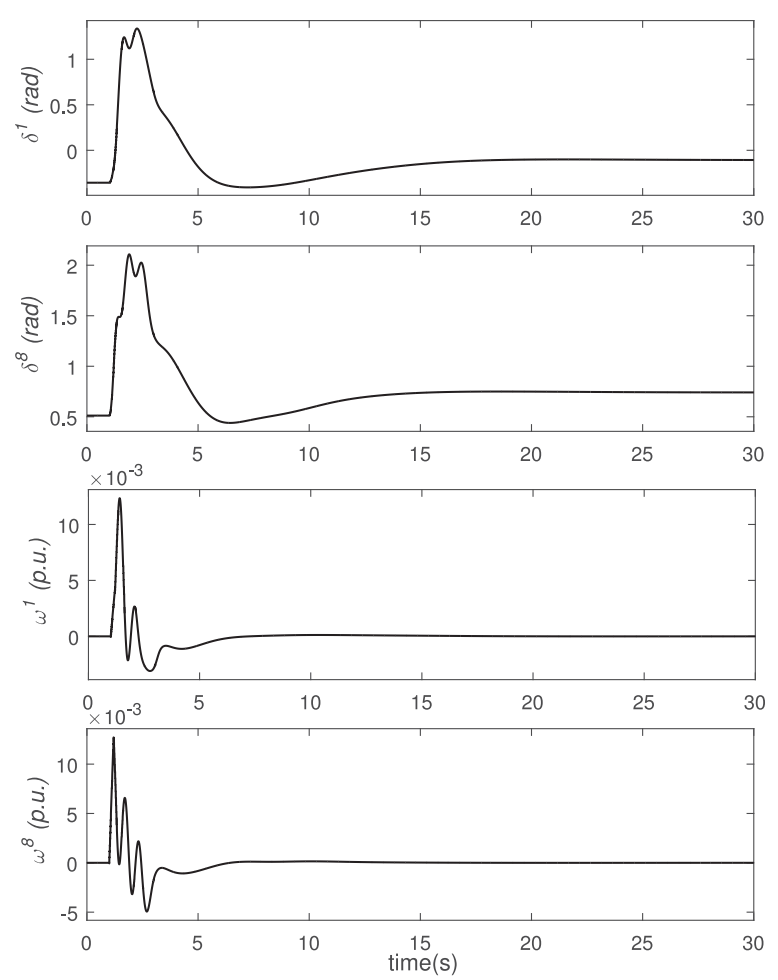

Fig. 8. $\delta$ and $\omega$ for units 1 and 8 under nonlinear control for Case B.
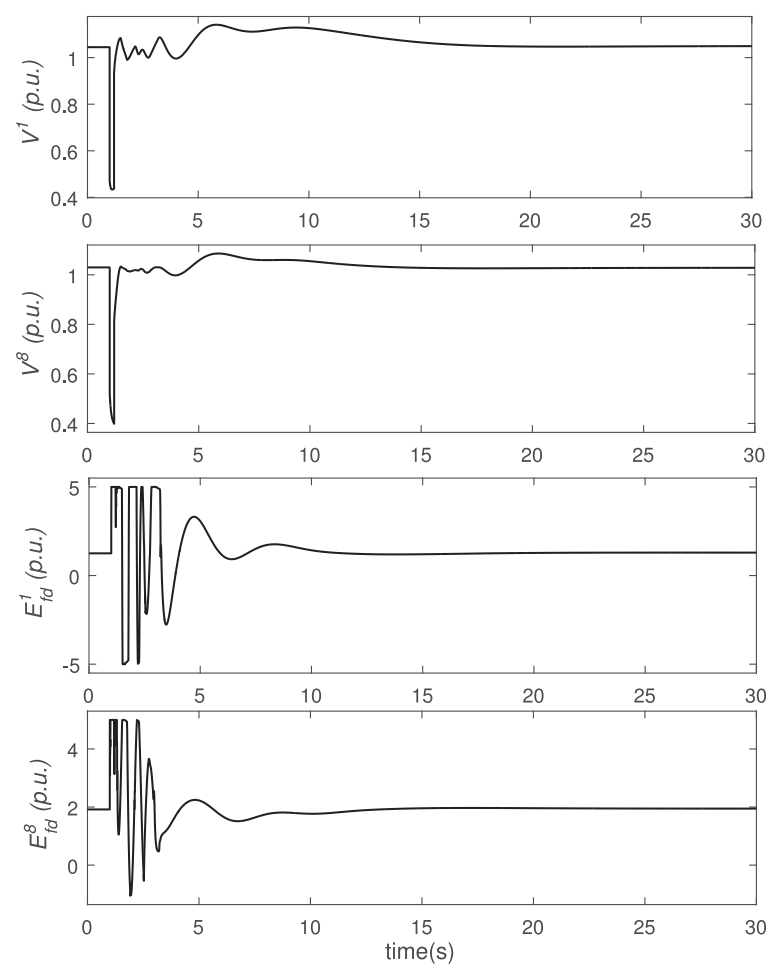

Fig. 9. $\quad V$ and $E_{f d}$ for units 1 and 8 under nonlinear control for Case B. disturbance (generators 1 to 8 ), as opposed to the generators which are not close (such as generator 13).

PSS and ELQR take longer time to settle down in case of a small disturbance (Case A), and fail to maintain the stability of the system in case of a large disturbance (Case B). This is because the control input from PSS and ELQR is not large enough
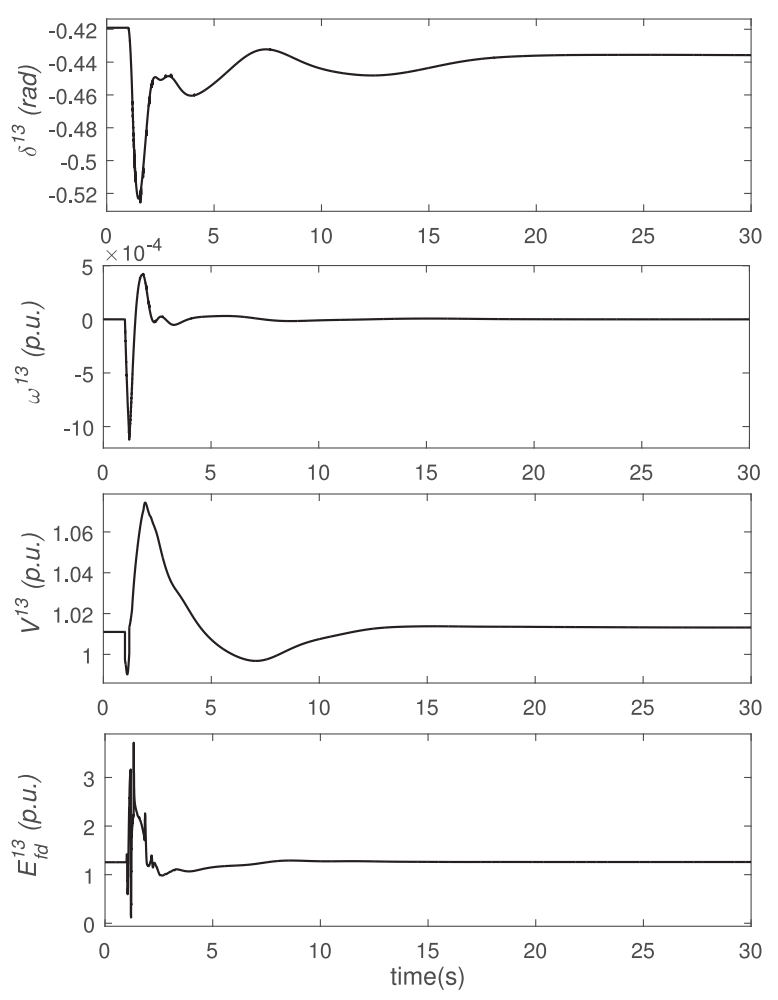

Fig. 10. Performance of unit 13 under nonlinear control for Case B.

after the disturbance, and the magnitude of the control input 756 is more-or-less uniform for all the generators (instead of being 757 larger for generators electrically closer to the disturbance and 758 smaller for machines which are electrically farther), as can be 759 observed in Figs. 4, 5, 9, and 10. If larger gains are used for PSS 760 and ELQR then they would also provide large control inputs. 761 But as PSS and ELQR are derived using linear model of power 762 systems and are designed specifically for small disturbances, 763 they may not work for large disturbances since a linear model is 764 not valid for large disturbances. Thus, using large gains for PSS 765 and ELQR (or other tools for small signal stability) may worsen 766 the situation in case of a large disturbance, or jeopardize the sta- 767 bility of the system even in case of a small disturbance. As the 768 proposed nonlinear control is derived specifically for nonlinear 769 model of power systems, it is not dependent on a particular equi- 770 librium point or a linear model evaluated at that point. Hence, 771 it ensures that both the small signal stability and the transient 772 stability of the system are maintained irrespective of whether 773 the disturbance is small or large.

Computational feasibility: The complete simulation of the power system, along with the dynamic estimators and the nonlinear controllers at each of the 16 machines, runs in real-time. In the case study, a $30 \mathrm{~s}$ simulation takes an average running time of $10.1 \mathrm{~s}$ on a personal computer with Intel Core 2 Duo, 2.0 $\mathrm{GHz}$ CPU and 2 GB RAM. Hence the computational requirements at one machine can be easily met for both estimation and control.

\section{CONCLUSION}

A nonlinear control scheme has been proposed for decen- 784 tralized control of power system dynamics. A normal form 785 
has been developed for modelling the subtransient dynamics of power systems, and the proposed control scheme has been subsequently derived using this normal form. Asymptotic stability of the whole system under the proposed control has been proved. It has been demonstrated using simulations that the method can be used to ensure both transient stability and small signal stability. Thus, it is a robust tool for mitigation of blackouts which are caused by poorly damped oscillations and other transient instabilities occurring in power systems.

\section{REFERENCES}

[1] P. Kundur et al., "Definition and classification of power system stability: IEEE/CIGRE joint task force on stability terms and definitions," IEEE Trans. Power Syst., vol. 19, no. 3, pp. 1387-1401, Aug. 2004.

[2] M. A. Pai and P. W. Sauer, Power System Dynamics and Stability. Englewood Cliffs, NJ, USA: Prentice-Hall, 1998, ch. 6.

[3] K. R. Padiyar, Power System Dynamics: Stability and Control. Tunbridge Wells, U.K.: Anshan Limited, 2004.

[4] B. Pal and B. Chaudhuri, Robust Control in Power Systems. New York, NY, USA: Springer, 2005, ch. 4.

[5] D. N. Kosterev, C. W. Taylor, and W. A. Mittelstadt, "Model validation for the August 10, 1996 WSCC system outage," IEEE Trans. Power Syst., vol. 14, no. 3, pp. 967-979, Aug. 1999.

[6] N. Kakimoto, A. Nakanishi, and K. Tomiyama, "Instability of interarea oscillation mode by autoparametric resonance," IEEE Trans. Power Syst., vol. 19, no. 4, pp. 1961-1970, Nov. 2004.

[7] Q. Lu, Y. Z. Sun, and S. Mei, Nonlinear Control Systems and Power System Dynamics. Dordrecht, The Netherlands: Springer, 2013.

[8] M. A. Mahmud, H. R. Pota, M. Aldeen, and M. J. Hossain, "Partial feedback linearizing excitation controller for multimachine power systems to improve transient stability," IEEE Trans. Power Syst., vol. 29, no. 2, pp. 561-571, Mar. 2014.

[9] W. Yao, L. Jiang, J. Fang, J. Wen, and S. Cheng, "Decentralized nonlinear optimal predictive excitation control for multi-machine power systems," Int. J. Elect. Power Energy Syst., vol. 55, pp. 620-627, 2014.

[10] Y. Guo, D. J. Hill, and Y. Wang, "Global transient stability and voltage regulation for power systems," IEEE Trans. Power Syst., vol. 16, no. 4, pp. 678-688, Nov. 2001.

[11] Q. Lu, Y. Z. Sun, Z. Xu, and T. Mochizuki, "Decentralized nonlinear optimal excitation control," IEEE Trans. Power Syst., vol. 11, no. 4, pp. 19571962, Nov. 1996

[12] J. W. Chapman, M. D. Ilic, C. A. King, L. Eng, and H. Kaufman, "Stabilizing a multimachine power system via decentralized feedback linearizing excitation control," IEEE Trans. Power Syst., vol. 8, no. 3, pp. 830-839, Aug. 1993.

[13] H. Liu, Z. Hu, and Y. Song, "Lyapunov-based decentralized excitation control for global asymptotic stability and voltage regulation of multi-machine power systems," IEEE Trans. Power Syst., vol. 27, no. 4, pp. 2262-2270, Nov. 2012.

[14] R. Yan, Z. Y. Dong, T. K. Saha, and R. Majumder, "A power system nonlinear adaptive decentralized controller design," Automatica, vol. 46, no. 2, pp. 330-336, Feb. 2010.

[15] Y. Wang, D. Cheng, C. Li, and Y. Ge, "Dissipative Hamiltonian realization and energy-based L2-disturbance attenuation control of multimachine power systems," IEEE Trans. Autom. Control vol. 48, no. 8, pp. 14281433, Aug. 2003.

[16] Q. Lu, S. Mei, W. Hu, F. F. Wu, Y. Ni, and T. Shen, "Nonlinear decentralized disturbance attenuation excitation control via new recursive design for multimachine power systems," IEEE Trans. Power Syst., vol. 16, no. 4, pp. 729-736, Nov. 2001.

[17] Y. Guo, D. J. Hill, and Y. Wang, "Nonlinear decentralized control of large-scale power systems," Automatica, vol. 36, no. 9, pp. 1275-1289, Sep. 2000.

[18] IEC Standard for Instrument Transformers, IEC Std 60044 ed. 1.2, 2003.

[19] IEEE Standard Requirements for Instrument Transformers, IEEE Std C57.13-2008 (Revision of IEEE Std C57.13-1993), Jul. 28, 2008.

[20] A. G. Phadke and J. S. Thorp, Synchronized Phasor Measurements and Their Applications. New York, NY, USA: Springer, 2008.

[21] IEEE Standard for Synchrophasor Measurements for Power Systems, IEEE Std C37.118.1-2011, Dec. 2011.
[22] IEEE Standard for Synchrophasor Measurements for Power SystemsAmendment 1: Modification of Selected Performance Requirements, IEEE Std C37.118.1a-2014 (Amendment to IEEE Std C37.118.1-2011), Apr. 2014.

[23] IEEE Guide for Synchronous Generator modelling Practices and Applications in Power System Stability Analyses, IEEE Std 1110-2002 (Revision of IEEE Std 1110-1991), 2003, pp. 1-72.

[24] J. J. Sanchez-Gasca et al., "Inclusion of higher order terms for small-signal (modal) analysis: Committee report-task force on assessing the need to include higher order terms for small-signal (modal) analysis," IEEE Trans. Power Syst., vol. 20, no. 4, pp. 1886-1904, Nov. 2005.

[25] A. Isidori, Nonlinear Control Systems. London, U.K.: Springer, 1995.

[26] P. Kundur, Power System Stability and Control. Noida, India: McGrawHill, 1994.

[27] Katsuhiko Ogata, Modern Control Engineering, 4th ed. Upper Saddle River, NJ, USA: Prentice-Hall, 2001.

[28] E. Ghahremani and I. Kamwa, "Local and wide-area PMU-based decentralized dynamic state estimation in multi-machine power systems," IEEE Trans. Power Syst., vol. 31, no. 1, pp. 547-562, Jan. 2016.

[29] Y. Cui and R. Kavasseri, "A particle filter for dynamic state estimation in multi-machine systems with detailed models," IEEE Trans. Power Syst., vol. 30, no. 6, pp. 3377-3385, Nov. 2015.

[30] K. Emami, T. Fernando, H. H. C. Iu, H. Trinh, and K. P. Wong, "Particle filter approach to dynamic state estimation of generators in power systems," IEEE Trans. Power Syst., vol. 30, no. 5, pp. 2665-2675, Sep. 2015.

[31] A. K. Singh and B. C. Pal, "Decentralized dynamic state estimation in power systems using unscented transformation," IEEE Trans. Power Syst., vol. 29, no. 2, pp. 794-804, Mar. 2014.

[32] N. Zhou, D. Meng, and S. Lu, "Estimation of the dynamic states of synchronous machines using an extended particle filter," IEEE Trans. Power Syst., vol. 28, no. 4, pp. 4152-4161, Nov. 2013.

[33] E. Scholtz, V. D. Donde, and J. C. Tournier, "Parallel computation of dynamic state estimation for power system," U.S. Patent 13/832 670, Mar $15,2013$.

[34] S. Julier, J. Uhlmann, and H. F. Durrant-Whyte, "A new method for the nonlinear transformation of means and covariances in filters and estimators," IEEE Trans. Autom. Control, vol. 45, no. 3, pp. 477-482, Mar. 2000

[35] A. K. Singh and B. C. Pal, "Report on the 68-bus, 16-machine, 5-area system," IEEE PES Task Force on Benchmark Systems for Stability Controls, Ver. 3.3, 2013.

[36] A. K. Singh and B. C. Pal, "An extended linear quadratic regulator for LTI systems with exogenous inputs," Automatica, vol. 76, pp. 10-16, Feb. 2017.

[37] A. K. Singh and B. C. Pal, "Decentralized control of oscillatory dynamics in power systems using an extended LQR," IEEE Trans. Power Syst., vol. 31, no. 3, pp. 1715-1728, May 2016.

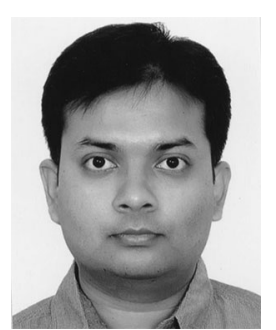

Abhinav Kumar Singh (S'12-M'15) received the B.Tech. degree from the Indian Institute of Technology, New Delhi, India, and the Ph.D. degree from Imperial College London, London, U.K., in August 2010 and January 2015, respectively, all in electrical engineering.

He is currently a Lecturer of Electrical Engineering at University of Lincoln, U.K., and a Visiting Research Fellow at Imperial College London, U.K His research interests include estimation, control, and communication aspects of power systems.

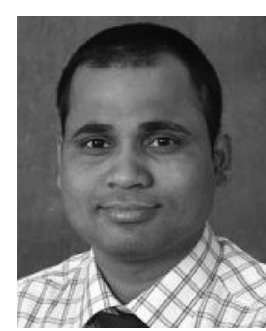

Bikash C. Pal (M'00-SM'02-F'13) received the B.E.E. (Hons.) degree from Jadavpur University, Kolkata, India, the M.E. degree from the Indian Institute of Science, Bangalore, India, and the Ph.D degree from Imperial College London, London, U.K. in 1990, 1992, and 1999, respectively, all in electrical engineering.

$\mathrm{He}$ is currently a Professor with the Department of Electrical and Electronic Engineering, Imperial College London. His current research interests include state estimation and power system dynamics.

Dr. Pal is the Editor-in-Chief of the IEEE TRANSACTIONS ON SUSTAINABLE ENERGY and a Fellow of the IEEE for his contribution to power system stability and control. 